\title{
Nonnegatively and Positively curved manifolds
}

\author{
Burkhard Wilking
}

The aim of this paper is to survey some results on nonnegatively and positively curved Riemannian manifolds. One of the important features of lower curvature bounds in general is the invariance under taking Gromov Hausdorff limits. Celebrated structure and finiteness results provide a partial understanding of the phenomena that occur while taking limits. These results however are not the subject of this survey since they are treated in other surveys of this volume.

In this survey we take the more classical approach and focus on "effective" results. There are relatively few general "effective" structure results in the subject. By Gromov's Betti number theorem the total Betti number of a nonnegatively curved manifold is bounded above by an explicit constant which only depends on the dimension. The Gromoll Meyer theorem says that a positively curved open manifold is diffeomorphic to the Euclidean space. In the case of nonnegatively curved open manifolds, the soul theorem of Cheeger and Gromoll and Perelman's solution of the soul conjecture clearly belong to the greatest structure results in the subject, as well.

Also relatively good is the understanding of fundamental groups of nonnegatively curved manifolds. A theorem of Synge asserts that an even dimensional orientable compact manifold of positive sectional curvature is simply connected. An odd dimensional positively curved manifold is known to be orientable (Synge), and its fundamental group is finite by the classical theorem of Bonnet and Myers. The fundamental groups of nonnegatively curved manifolds are virtually abelian, as a consequence of Toponogov's splitting theorem. However, one of the "effective" conjectures in this context, the so called Chern conjecture, was refuted: Shankar [1998] constructed a positively curved manifold with a non cyclic abelian fundamental group.

As we will discuss in the last section the known methods for constructing nonnegatively curved manifolds are somewhat limited. The most important tools are the O'Neill formulas which imply that the base of a Riemannian submersion has nonnegative (positive) sectional curvature if the total space has. We recall that a smooth surjective map $\sigma: M \rightarrow B$ between two Riemannian manifolds is called a Riemannian submersion if the dual 
$\sigma_{*}^{a d}: T_{\sigma(p)} B \rightarrow T_{p} M$ of the differential of $\sigma$ is length preserving for all $p \in M$. Apart from taking products, the only other method is a special glueing technique, which was used by Cheeger, and more recently by Grove and Ziller to construct quite a few interesting examples of nonnegatively curved manifolds.

By comparing with the class of known positively curved manifolds, the nonnegatively curved manifolds form a huge class. In fact in dimensions above 24 all known simply connected compact positively curved manifolds are diffeomorphic to rank 1 symmetric spaces. Due to work of the author the situation is somewhat better in the class of known examples of manifolds with positive curvature on open dense sets, see section 4 .

Given the drastic difference in the number of known examples, it is somewhat painful that the only known obstructions on positively curved compact manifolds, which do not remain valid for the nonnegatively curved manifolds, are the above quoted results of Synge and Bonnet Myers on the fundamental groups.

Since the list of general structure results is not far from being complete by now, the reader might ask why a survey on such a subject is necessary. The reason is that there are a lot of other beautiful theorems in the subject including structure results, but they usually need additional assumptions.

We have subdivided the paper in five sections. Section 1 is on sphere theorems and related rigidity results some notes on very recent significant developments were added in proof and can be found in section 6 . In section 2 , we survey results on compact nonnegatively curved manifolds, and in section 3 , results on open nonnegatively curved manifolds. Then follows a section on compact positively curved manifolds with symmetry, since this was a particularly active area in recent years. Although we pose problems and conjectures throughout the paper we close the paper with a section on open problems.

We do not have the ambition to be complete or to sketch all the significant historical developments that eventually led to the stated results. Instead we will usually only quote a few things according to personal taste.

\section{Sphere theorems and related rigidity results}

A lot of techniques in the subject were developed or used in connection with proving sphere theorems. In this section we survey some of these results. We recall Toponogov's triangle comparison theorem. Let $M$ be a complete manifold with sectional curvature $K \geq \kappa$ and consider a geodesic triangle $\Delta$ in $M$ consisting of minimal geodesics with length $a, b, c \in \mathbb{R}$. Then there exists a triangle in the 2-dimensional complete surface $M_{\kappa}^{2}$ of constant curvature $\kappa$ with side length $a, b, c$ and the angles in the comparison triangle bound the corresponding angles in $\Delta$ from below.

1.1. Topological sphere theorems. We start with the classical sphere theorem of Berger and Klingenberg. 
TheOREM 1.1 (Quarter pinched sphere theorem). Let $M$ be a complete simply connected manifold with sectional curvature $1 / 4<K \leq 1$. Then $M$ is homeomorphic to the sphere.

The proof has two parts. The first part is to show that the injectivity radius of $M$ is at least $\pi / 2$. This is elementary in even dimensions. In fact by Synge's Theorem any even dimensional oriented manifold with curvature $0<K \leq 1$ has injectivity radius $\geq \pi$. In odd dimensions the result is due to Klingenberg and relies on a more delicate Morse theory argument on the loop space.

The second part of the proof is due to Berger. He showed that any manifold with injectivity radius $\geq \pi / 2$ and curvature $>1$ is homeomorphic to a sphere. In fact by applying Toponogov's theorem to two points of maximal distance, he showed that the manifold can be covered by two balls, which are via the exponential map diffeomorphic to balls in the Euclidean space.

Grove and Shiohama [1977] gave a significant improvement of Berger's theorem, by replacing the lower injectivity radius bound by a lower diameter bound.

Theorem 1.2 (Diameter sphere theorem). Any manifold with sectional curvature $\geq 1$ and diameter $>\pi / 2$ is homeomorphic to a sphere.

More important than the theorem was the fact the proof introduced a new concept: critical points of distance functions. A point $q$ is critical with respect to the distance function $d(p, \cdot)$ if the set of initial vectors of minimal geodesics from $q$ to $p$ intersect each closed half space of $T_{q} M$. If the point $q$ is not critical it is not hard to see that there is a gradient like vectorfield $X$ in a neighborhood of $q$. A vectorfield is said to be gradient like if for each integral curve $c$ of $X$ the map $t \mapsto d(p, c(t))$ is a monotonously increasing bilipschitz map onto its image. An elementary yet important observation is that local gradient like vectorfields can be glued together using a partition of unity.

Proof of the DiAmeter SPHERe theorem. We may scale the manifold such that its diameter is $\pi / 2$ and the curvature is strictly $>1$. Choose two points $p, q$ of maximal distance $\pi / 2$, and let $z$ be an arbitrary third point. Consider the spherical comparison triangle $(\tilde{p}, \tilde{q}, \tilde{z})$. We do know that the side length of $(\tilde{p}, \tilde{z})$ and $(\tilde{q}, \tilde{z})$ are less or equal to $\pi / 2$ whereas $d_{\mathbb{S}^{2}}(\tilde{p}, \tilde{q})=\pi / 2$. This implies that the angle of the triangle at $\tilde{z}$ is $\geq \pi / 2$. By Toponogov's theorem any minimal geodesic triangle with corners $p, q, z$ in $M$ has an angle strictly larger than $\pi / 2$ based at $z$. This in turn implies that the distance function $d(p, \cdot)$ has no critical points in $M \backslash\{p, q\}$. Thus there is a gradient like vectorfield $X$ on $M \backslash\{p, q\}$. Furthermore without loss of generality $X$ is given on $B_{r}(p) \backslash\{p\}$ by the actual gradient of the distance function $d(p, \cdot)$, where $r$ is smaller than the injectivity radius. We may also assume $\|X(z)\| \leq d(q, z)^{2}$ for all $z \in M \backslash\{p, q\}$. Then the flow $\Phi$ of $X$ exists for all 
future times and we can define a diffeomorphism

$$
\psi: T_{p} M \rightarrow M \backslash\{q\}
$$

as follows: for a unit vector $v \in T_{p} M$ and a nonnegative number $t$ put $\psi(t \cdot v)=\exp (t v)$ if $t \in[0, r]$ and $\psi(t \cdot v)=\Phi_{t-r}(\exp (r v))$ if $t \geq r$. Clearly this implies that $M$ is homeomorphic to a sphere.

There is another generalization of the sphere theorem of Berger and Klingenberg. A manifold is said to have positive isotropic curvature if for all orthonormal vectors $e_{1}, e_{2}, e_{3}, e_{4} \in T_{p} M$ the curvature operator satisfies

$R\left(e_{1} \wedge e_{2}+e_{3} \wedge e_{4}, e_{1} \wedge e_{2}+e_{3} \wedge e_{4}\right)+R\left(e_{1} \wedge e_{3}+e_{4} \wedge e_{2}, e_{1} \wedge e_{3}+e_{4} \wedge e_{2}\right)>0$

By estimating the indices of minimal 2 spheres in a manifold of positive isotropic curvature, Micallef and Moore [1988] were able to show that

THEOREM 1.3. A simply connected compact Riemannian manifold of positive isotropic curvature is a homotopy sphere.

A simple computation shows that pointwise strictly quarter pinched manifolds have positive isotropic curvature. Thus the theorem of Micallef and Moore is a generalization of the quarter pinched sphere theorem. A more direct improvement of the quarter pinched sphere theorem is due to Abresch and Meyer [1996].

THEOREM 1.4. Let $M$ be a compact simply connected manifold with sectional curvature $\frac{1}{4\left(1+10^{-6}\right)^{2}} \leq K \leq 1$. Then one of the following holds

- $M$ is homeomorphic to a sphere.

- $n$ is even and the cohomology ring $H^{*}\left(M, \mathbb{Z}_{2}\right)$ is generated by one element.

It is a well known result in topology that the $\mathbb{Z}_{2}$ cohomology rings of spaces which are generated by one element are precisely given by the $\mathbb{Z}_{2}$-cohomology rings of rank 1 symmetric spaces $\mathbb{R} \mathbb{P}^{n}, \mathbb{C P}^{n}, \mathbb{H} \mathbb{P}^{n}, \mathrm{CaP}^{2}$ and $\mathbb{S}^{n}$, cf. [Zhizhou, 2002].

The proof of Theorem 1.4 has again two parts. Abresch and Meyer first establish that the injectivity radius of $M$ is bounded below by the conjugate radius which in turn is bounded below by $\pi$. From the diameter sphere theorem it is clear that without loss of generality $\operatorname{diam}(M, g) \leq \pi\left(1+10^{-6}\right)$. They then establish the horse shoe inequality, which was conjectured by Berger: for $p \in M$ and any unit vector $v \in T_{p} M$ one has

$$
d(\exp (\pi v), \exp (-\pi v))<\pi
$$

In particular $\exp (\pi v)$ and $\exp (-\pi v)$ can be joined by a unique minimal geodesic. Once the horse shoe inequality is established it is easy to see that there is a smooth map $f: \mathbb{R P}^{n} \rightarrow M^{n}$ such that in odd dimensions the 
integral degree is 1 and in even dimensions the $\mathbb{Z}_{2}$-degree is 1 . The theorem then follows by a straightforward cohomology computation.

The horse shoe inequality relies on a mixed Jacobi field estimate. We only state the problem here in a very rough form. Let $c$ be a normal geodesic in $M$ and $J$ a Jacobifield with $J(0)=0$. Suppose that at time $t_{0}=\frac{2 \pi}{3}$ the value $\left\|J\left(t_{0}\right)\right\|$ is quite a bit smaller than one would expect by Rauch's comparison from the lower curvature bound. Can one say that $\|J(t)\|$ is also quite a bit smaller than in Rauch's comparison for $t \geq\left[t_{0}, \pi\right]$ ? Abresch and Meyer gave an affirmative answer. If one wants to improve the pinching constant one certainly needs to improve their Jacobifield estimate.

1.2. Differentiable sphere theorems. It is not known whether there are exotic spheres with positive sectional curvature. A closely related question is whether one can improve in any (or all) of the above mentioned topological sphere theorems the conclusion from homeomorphic to being diffeomorphic to a sphere. In other words, can one turn the topological sphere theorems into differentiable sphere theorems. In each case this is an open question. However, there are quite a few differentiable sphere theorems, which hold under stronger assumptions.

The first differentiable sphere theorem was established in his thesis by Gromoll. He had a pinching condition $\delta(n)<K \leq 1$ but his pinching constant $\delta(n)$ depended upon the dimension, i.e. $\delta(n) \rightarrow 1$ for $n \rightarrow \infty$.

Sugimoto and Shiohama [1971] established the first bound which was independent of the dimension with $\delta=0.87$. In a series of papers Grove, Im Hof, Karcher and Ruh obtained the following result

ThEOREM 1.5. There is a decreasing sequence of numbers $\delta(n)$ with $\lim _{n \rightarrow \infty} \delta(n)=0.68$ such that any simply connected manifold $(M, g)$ with $\delta(n)<K \leq 1$ is diffeomorphic to the sphere $\mathbb{S}^{n}$. Furthermore the diffeomorphism may be chosen such that the natural action $\operatorname{Iso}(M, g)$ on $M$ corresponds under $f$ to a linear action on $\mathbb{S}^{n}$.

If one does not insist on an equivariant diffeomorphisms, then the pinching constant can be improved somewhat. Suyama [1995] showed that a simply connected manifold with $0.654<K \leq 1$ is diffeomorphic to the sphere.

The work of Weiss [1993] goes in a different direction. He uses the fact that a quarter pinched sphere $M^{n}$ has Morse perfection $n$. A topological sphere $M^{n}$ is said that to have Morse perfection $\geq k$ if there is a smooth $\operatorname{map} \Psi: \mathbb{S}^{k} \rightarrow \mathbb{C}^{\infty}(M, \mathbb{R})$ satisfying $\Psi(-p)=-\Psi(p)$, and for each $p \in \mathbb{S}^{k}$ the function $\Psi(p)$ is a Morse function with precisely two critical points. It is not hard to see that a quarter pinched sphere has Morse perfection $n$. Weiss used this to rule out quite a few of the exotic spheres by showing that their Morse perfection is $<n$. He showed that in dimensions $n=4 m-1$ any exotic sphere bounding a parallelizable manifold has odd order in the group of exotic spheres unless the Morse perfection $\leq n-2$. 
By Hitchin there are also exotic spheres with a non-vanishing $\alpha$-invariant, and thus these spheres do not even admit metrics with positive scalar curvature, see the survey of Jonathan Rosenberg.

Similar to the quarter pinched sphere theorem, one can also strengthen the assumptions in the diameter sphere theorem in order to get a differentiable sphere theorem. This was carried out by Grove and Wilhelm [1997].

THEOREM 1.6. Let $M$ be an $n$-manifold with sectional curvature $\geq 1$ containing $(n-2)$-points with pairwise distance $>\pi / 2$. Then $M$ is diffeomorphic to a sphere.

If one has only $k$ points with pairwise distance $>\pi / 2$, then Grove and Wilhelm obtain restrictions on the differentiable structure of $M$.

With a slight variation of the proof of Grove and Wilhelm one can actually get a slightly better result. Let $M$ be an inner metric space. We say that $M$ has a weak 2 -nd packing radius $\geq r$ if $\operatorname{diam}(M) \geq r$. We say it has a weak $k$-th packing radius $\geq r$ if there is a point $p \in M$ such that $\partial B_{r}(p)$ is connected and endowed with its inner metric has weak $(k-1)$-th packing radius $\geq r$.

THEOREM 1.7. Let $(M, g)$ be an $n$-manifold with sectional curvature $\geq 1$ and weak $(k+1)$-th packing radius $>\pi / 2$. Then there is a family of metrics $g_{t}$ $\left(t \in[0,1)\right.$ with sectional curvature $\geq 1$ and $g_{0}=g$ such that $\left(M, g_{t}\right)$ converges for $t \rightarrow 1$ to an $n$-dimensional Alexandrov space $A$ satisfying: If $k \geq n$, then $A$ is isometric to the standard sphere. If $k<n$, then $A$ is given by the $k$-th iterated suspension $\Sigma^{k} A^{\prime}$ of an $n-k$-dimensional Alexandrov space $A^{\prime}$.

Corollary 1.8. Let $\varepsilon>0$. A manifold with sectional curvature $\geq 1$ and diameter $>\pi / 2$ also admits a metric with sectional curvature $\geq 1$ and diameter $>\pi-\varepsilon$.

As in the paper of Grove and Wilhelm, one can show in the situation of Theorem 1.7 that there is a sequence of positively metrics $\tilde{g}_{i}$ on the standard sphere with curvature $\geq 1$ such that $\left(\mathbb{S}^{n}, \tilde{g}_{i}\right)_{i \in \mathbb{N}}$ converges to $A$ as well. In particular, Grove and Wilhelm showed that an affirmative answer to the following question would imply the differentiable diameter sphere theorem.

Question 1.9 (Smooth stability conjecture). Suppose a sequence of compact $n$-manifolds $\left(M_{k}, g_{k}\right)$ with curvature $\geq-1$ converges in the Gromov Hausdorff topology to an n-dimensional compact Alexandrov space A. Does this imply that for all large $k_{1}$ and $k_{2}$ the manifolds $M_{k_{1}}$ and $M_{k_{2}}$ are diffeomorphic?

By Perelman's stability theorem it is known that $M_{k_{1}}$ and $M_{k_{2}}$ are homeomorphic for all large $k_{1}$ and $k_{2}$, see the article of Vitali Kapovitch in this volume. 
Sketch of The Proof of TheOREM 1.7. Let $p, q \in M$ be points such that $d(p, q)>\pi / 2+\varepsilon$ for some $\varepsilon>0$. We claim that we can find a continuous family of metrics with $g_{0}=g$ and $K_{t} \geq 1$ such that $\left(M, g_{t}\right)$ converges for $t \rightarrow 1$ to the suspension of $\partial B_{\pi / 2}(p)$.

We consider the suspension $X$ of $M$, i.e., $X=[-\pi / 2, \pi / 2] \times M / \sim$ where the equivalence classes of $\sim$ are given by $p_{+}:=\{\pi / 2\} \times M, p_{-}:=$ $\{-\pi / 2\} \times M$ and the one point sets $\{(t, p)\}$ for $|t| \neq \pi / 2$. Recall that $X$ endowed with the usual warped product metric is an Alexandrov space with curvature $\geq 1$.

We consider the curve $c(t)=((1-t) \pi / 2, p)$ as a curve in $X, r(t):=$ $\pi / 2+\varepsilon(1-t)$ and the ball $B_{r(t)}(c(t)) \subset X$. Put $N_{t}:=\partial B_{r(t)}(c(t))$. Since $X \backslash B_{r(t)}(c(t))$ is strictly convex and $N_{t}$ is contained in the Riemannian manifold $X \backslash\left\{p_{ \pm}\right\}$for all $t \neq 1$, it follows that $N_{t}$ is an Alexandrov space with curvature $\geq 1$ for all $t \in[0,1]$. Clearly $N_{0}$ is up to a small scaling factor isometric to $M$. Moreover $N_{1}$ is isometric to the suspension of $\partial B_{\pi / 2}(p) \subset M$.

Using that $N_{t}$ is strictly convex in the Riemannian manifold $X \backslash\left\{p_{ \pm}\right\}$for $t \in[0,1)$, it follows that the family $N_{t}$ can be approximated by a family of strictly convex smooth submanifolds $\tilde{N}_{t} \subset X \backslash\left\{p_{ \pm}\right\}, t \in[0,1)$. Furthermore, one may assume that $\lim _{t \rightarrow 1} N_{t}=N_{1}=\lim _{t \rightarrow 1} \tilde{N}_{t}$.

We found a family of metrics $g_{t}$ of curvature $>1$ such that $\left(M, g_{t}\right)$ converges to the suspension of $\partial B_{\pi / 2}(p)$. We may assume that $\partial B_{\pi / 2}(p)$ has weak $k$-th packing radius $>\pi / 2$ and $k \geq 2$.

We now choose a curve of points $q_{t} \in M$ converging for $t \rightarrow 1$ to a point on the equator $q_{1} \in \partial B_{\pi / 2}(p)$ of the limit space such that there is a point $q_{2}$ in $\partial B_{\pi / 2}(p)$ whose intrinsic distance to $q_{1}$ is $>\pi / 2$.

We now repeat the above construction for all $t \in(0,1)$ with $(M, g, p)$ replaced by $\left(M, g_{t}, q_{t}\right)$. This way we get for each $t$ an one parameter family of smooth metrics $g(t, s)$ with $K \geq 1$ which converges for $s \rightarrow 1$ to the suspension of the boundary of $B_{\pi / 2}\left(q_{t}\right) \subset\left(M, g_{t}\right)$. It is then easy to see that one can choose the metrics such that they depend smoothly on $s$ and $t$. Moreover, after a possible reparameterization of $g(s, t)$ the one parameter family $t \mapsto g(t, t)$ converges to the double suspension of the boundary of $B_{\pi / 2}\left(q_{1}\right) \subset$ $\partial B_{\pi / 2}(p)$. Clearly the theorem follows by iterating this process.

We recall that to each Riemannian manifold $(M, g)$ and each point $p \in M$ one can assign a curvature operator $R: \Lambda_{2} T_{p} M \rightarrow \Lambda_{2} T_{p} M$. We call the operator 2-positive if the sum of the smallest two eigenvalues is positive. It is known that manifolds with 2-positive curvature operator have positive isotropic curvature.

THEOREM 1.10. Let $(M, g)$ be a compact manifold with 2-positive curvature operator. Then the normalized Ricci flow evolves $g$ to a limit metric of constant sectional curvature.

In dimension 3 the theorem is due to Hamilton [1982]. Hamilton [1986] also showed that the theorem holds for 4-manifolds with positive curvature 
operator. This was extended by Chen to 4-manifolds with 2-positive curvature operator. In dimension 2 it was shown by Hamilton and Chow that for any surface the normalized Ricci flow converges to limit metric of constant curvature. In dimensions above 4 the theorem is due to Böhm and Wilking [2006]. For $n \geq 3$ the proof solely relies on the maximum principle and works more generally in the category of orbifolds.

We recall that a family of metrics $g_{t}$ on $M$ is said to be a solution of the Ricci flow if

$$
\frac{\partial}{\partial t} g_{t}=-2 \text { Ric }_{t}
$$

Hamilton showed that if one represents the curvature operator $R$ with respect to suitable moving orthonormal frames, then

$$
\frac{\partial}{\partial t} R=\Delta R+2\left(R^{2}+R^{\#}\right)
$$

where $R^{\#}=\operatorname{ad} \circ R \wedge R \circ \mathrm{ad}^{*}$, ad: $\Lambda_{2} \mathfrak{s o}\left(T_{p} M\right) \rightarrow \mathfrak{s o}\left(T_{p} M\right)$ is the adjoint representation and where we have identified $\Lambda_{2} T_{p} M$ with the Lie algebra $\mathfrak{s o}\left(T_{p} M\right)$. Hamilton's maximum principle allows to deduce certain dynamical properties of the PDE from dynamical properties of the ODE

$$
\frac{d}{d t} R=R^{2}+R^{\#}
$$

Sketch of the Proof of Theorem 1.10. We let $S_{B}^{2}(\mathfrak{s o}(n))$ denote the vectorspace of algebraic curvature operators satisfying the Bianchi identity.

We call a continuous family $C(s)_{s \in[0,1)} \subset S_{B}^{2}(\mathfrak{s o}(n))$ of closed convex $\mathrm{O}(n)$-invariant cones of full dimension a pinching family, if

(1) each $\mathrm{R} \in C(s) \backslash\{0\}$ has positive scalar curvature,

(2) $\mathrm{R}^{2}+\mathrm{R}^{\#}$ is contained in the interior of the tangent cone of $C(s)$ at $\mathrm{R}$ for all $\mathrm{R} \in C(s) \backslash\{0\}$ and all $s \in(0,1)$,

(3) $C(s)$ converges in the pointed Hausdorff topology to the onedimensional cone $\mathbb{R}^{+} I$ as $s \rightarrow 1$.

The argument in [Böhm and Wilking, 2006] has two parts. One part is a general argument showing for any pinching family $C(s)(s \in[0,1))$ that on any compact manifold $(M, g)$ for which the curvature operator is contained in the interior of $C(0)$ at every point the normalized Ricci flow evolves $g$ to a constant curvature limit metric. In the proof of this result one first constructs to such a pinching family a pinching set in the sense Hamilton which in turn gives the convergence result.

The harder problem is actually to construct a pinching family with $C(0)$ being the cone of 2-nonnegative curvature operators. Here a new tool is established. It is a formula that describes how this ordinary differential equation $R^{\prime}=R^{2}+R^{\#}$ changes under $\mathrm{O}(n)$-equivariant linear transformations. By chance the transformation law is a lot simpler than for a generic $\mathrm{O}(n)$-invariant quadratic expression. The transformation law often allows to construct new ODE-invariant curvature cones as the image of a given 
invariant curvature cone under suitable equivariant linear transformation $l: S_{B}^{2}(\mathfrak{s o}(n)) \rightarrow S_{B}^{2}(\mathfrak{s o}(n))$. This in turn is used to establish the existence of a pinching family.

1.3. Related rigidity results. We first mention the diameter rigidity theorem of Gromoll and Grove [1987]

THEOREM 1.11 (Diameter rigidity). Let $(M, g)$ be a compact manifold with sectional curvature $K \geq 1$ and diameter $\geq \pi / 2$. Then one of the following holds:

a) $M$ is homeomorphic to a sphere.

b) $M$ is locally isometric to a rank one symmetric space.

The original theorem allowed a potential exceptional case

- $M$ has the cohomology ring of the Cayley plane, but is not isometric to the Cayley plane.

This case was ruled out much later by the author, see [Wilking, 2001].

The proof of the diameter rigidity theorem is closely linked to the rigidity of Hopf fibrations which was established by Gromoll and Grove [1988] as well

TheOREM 1.12 (Rigidity of Hopf fibrations). Let $\sigma: \mathbb{S}^{n} \rightarrow B$ be a Riemannian submersion with connected fibers. Then $\sigma$ is metrically congruent to a Hopf fibration. In particular the fibers are totally geodesic and $B$ is rank one symmetric space.

Similarly to the previous theorem, the original theorem allowed for a possible exception, Grove and Gromoll assumed in addition $(n, \operatorname{dim} B) \neq(15,8)$. Using very different methods, the rigidity of this special case was proved by the author in [Wilking, 2001]. This in turn ruled out the exceptional case in the diameter rigidity theorem as well.

Sketch of The PRoOf of THE DiAmeter RIGIDITy THEOREM. The proof of the diameter rigidity theorem is the most beautiful rigidity argument in positive curvature. One assumes that the manifold is not homeomorphic to a sphere. Let $p$ be a point with $N_{2}:=\partial B_{\pi / 2}(p) \neq \emptyset$. One defines $N_{1}=\partial B_{\pi / 2}\left(N_{2}\right)$ as the boundary of the distance tube $B_{\pi / 2}\left(N_{2}\right)$ around $N_{2}$. It then requires some work to see that $N_{1}$ and $N_{2}$ are totally geodesic submanifolds without boundary satisfying $N_{2}=\partial B_{\pi / 2}\left(N_{1}\right)$.

Not both manifolds can be points, since otherwise one can show that $M$ is homeomorphic to a sphere. If one endows the unit normal bundle $\nu^{1}\left(N_{i}\right)$ with its natural connection metric, then Grove and Gromoll show in a next step that the map $\sigma_{i}: \nu^{1}\left(N_{i}\right) \rightarrow N_{j}, v \mapsto \exp (\pi / 2 v)$ is a Riemannian submersion, $\{i, j\}=\{1,2\}$. Furthermore $\sigma_{i}$ restricts to a Riemannian submersion $\nu_{q}^{1}\left(N_{i}\right) \rightarrow N_{j}$ for all $q \in N_{i}$. 
In the simply connected case one shows that $N_{i}$ is simply connected as well, $i=1,2$. By the rigidity of submersions defined on Euclidean spheres (Theorem 1.12) we deduce that $N_{i}$ is either a point or a rank one symmetric space with diameter $\pi / 2$. Going back to the definition of $N_{1}$, it is then easy to see that $N_{1}=\{p\}$. Using that $\sigma_{1}: \mathbb{S}^{n-1} \rightarrow N_{2}$ is submersion with totally geodesic fibers, one can show that the pull back metric $\exp _{p}^{*} g$ on $B_{\pi / 2}(0) \subset T_{p} M$ is determined by $\sigma_{1}$. Thus $M$ is isometric to a rank one symmetric space.

In the non simply connected case one can show that either the universal cover is not a sphere and thereby symmetric or $\operatorname{dim}\left(N_{1}\right)+\operatorname{dim}\left(N_{2}\right)=n-1$. In the latter case it is not hard to verify that $M$ has constant curvature one.

Since the proof of the differentiable sphere theorem for manifolds with 2-positive curvature follows from a Ricci flow argument it is of course not surprising that it has a rigidity version as well.

THEOREM 1.13. A simply connected compact manifold with 2-nonnegative curvature operator satisfies one of the following statements.

- The normalized Ricci flow evolves the metric to a limit metric which is up to scaling is isometric to $\mathbb{S}^{n}$ or $\mathbb{C P}^{n / 2}$.

- $M$ is isometric to an irreducible symmetric space.

- $M$ is isometric to nontrivial Riemannian product.

Of course in the last case the factors of $M$ have nonnegative curvature operators. By Theorem $2.2(M, g)$ admits a possibly different metric $g_{1}$ such that $\left(M, g_{1}\right)$ is locally isometric to $(M, g)$ and $\left(M, g_{1}\right)$ is finitely covered by a Riemannian product $T^{d} \times M^{\prime}$ where $M^{\prime}$ is simply connected and compact. This effectively gives a reduction to the simply connected case.

The theorem has many names attached to it. Of course Theorem 1.10 (Hamilton [1982,1986], Böhm and Wilking [2006]) enters as the 'generic' case. This in turn was used by $\mathrm{Ni}$ and $\mathrm{Wu}$ [2006] to reduce the problem from 2-nonnegative curvature operators to nonnegative curvature operators. One has to mention Gallot and Meyer's [1975] investigation of manifolds with nonnegative curvature operator using the Bochner technique. Berger's classification of holonomy groups, as well as Mori's [1979], Siu and Yau's [1980] solution of the Frankel conjecture are key tools. Based on this Chen and Tian [2006] proved convergence of the Ricci flow for compact Kähler manifolds with positive bisectional curvature.

Sketch of A Proof of Theorem 1.13. Consider first the case that the curvature operator of $M$ is not nonnegative. We claim that then the Ricci flow immediately evolves $g$ to a metric with 2-positive curvature operator.

We consider a short time solution $g(t)$ of the Ricci flow and let $f:[0, \varepsilon) \times$ $M \rightarrow \mathbb{R}$, denote the function which assigns to $(t, p)$ the sum of the lowest two eigenvalues of the curvature operator of $(M, g(t))$ at $p$. We first want to 
show that $f(t, \cdot)$ is positive somewhere for small $t>0$. We may assume that $f(0, p)=0$ for all $p$. It is straightforward to check that $f$ satisfies

$$
\left.\frac{\partial f}{\partial t}\right|_{t=0_{+}}(0, p) \geq q(R):=\left.\frac{\partial}{\partial t}\right|_{t=0_{+}}\left(\lambda_{1}+\lambda_{2}\right)\left(R+t\left(R^{2}+R^{\#}\right)\right) .
$$

From the invariance of 2-nonnegative curvature operators it is known that $q(R) \geq 0$. In fact a detailed analysis of the proof shows that $q(R) \geq$ $2\left(\lambda_{1}(R)\right)^{2}$. In the present situation we deduce by a first order argument that $f(t, p)$ becomes positive somewhere for small $t>0$. Now it is not hard to establish a strong maximum principle that shows that $f(t, \cdot)$ is everywhere positive for small $t>0$, see $\mathrm{Ni}$ and $\mathrm{Wu}$ [2006]. In other words $\left(M, g_{t}\right)$ has 2-positive curvature operator for $t>0$ and the result follows from Theorem 1.10.

We are left with the case that the curvature operator of $(M, g)$ is nonnegative. Essentially this case was already treated by Gallot and Meyer using the Bochner technique, see [Petersen, 2006]. We present a slightly different argument following Chow and Yang (1989). Using Hamilton's [1986] strong maximum principle one deduces that for $t>0$ the curvature operator of $\left(M, g_{t}\right)$ has constant rank and that the kernel is parallel. Thus either $\mathrm{R}_{t}$ is positive or the holonomy is non generic. We may assume that $M$ does not split as a product. Hence without loss of generality $M$ is irreducible with non generic holonomy. Since $\left(M, g_{t}\right)$ clearly has positive scalar curvature Berger's classification of holonomy groups implies that $\operatorname{Hol}(M) \cong \mathrm{U}(n / 2), \operatorname{Sp}(1) \operatorname{Sp}(n / 4)$ unless $(M, g)$ is a symmetric space. In the case of $\operatorname{Hol}(M) \cong \mathrm{Sp}(1) \operatorname{Sp}(n)$ we can employ another theorem of Berger [1966] to see that $M$ is up to scaling isometric to $\mathbb{H} \mathbb{P}^{n / 4}$, since in our case the sectional curvature of $\left(M, g_{t}\right)$ is positive. In the remaining case $\operatorname{Hol}(M)=\mathrm{U}(n / 2)$ it follows that $M$ is Kähler and $\left(M, g_{t}\right)$ has positive (bi-)sectional curvature. By Mori [1979] and Siu and Yau's [1980] solution of the Frankel conjecture $M$ is biholomorphic to $\mathbb{C} \mathbb{P}^{n / 2}$. In particular, $M$ admits a Kähler Einstein metric. Due to work of Chen and Tian [2006] it follows, that the normalized Ricci flow on $M$ converges to the Fubini study metric which completes the proof.

\section{Compact nonnegatively curved manifolds}

The most fundamental obstruction to this date is Gromov's Betti number theorem.

TheOREm 2.1 (Gromov, 1981). Let $M^{n}$ be an $n$-dimensional complete manifold with nonnegative sectional curvature, and let $\mathbb{F}$ be a field. Then the total Betti number satisfies

$$
b(M, \mathbb{F}):=\sum_{i=0}^{n} b_{i}(M, \mathbb{F}) \leq 10^{10 n^{4}} .
$$


Gromov's original bound on the total Betti number was depending double exponentially on the dimension. The improvement is due to Abresch [1987]. However, this bound is not optimal either. In fact Gromov posed the problem whether the best possible bound is $2^{n}$, the total Betti number of the $n$-dimensional torus. The statement is particularly striking since the nonnegatively curved manifolds in a fixed dimension $\geq 7$ have infinitely many homology types with respect to integer coefficients. More generally Gromov gave explicit estimates for the total Betti numbers of compact $n$-manifolds with curvature $\geq-1$ and diameter $\leq D$. The proof is an ingenious combination of Toponogov's theorem and critical point theory.

Sketch of the Proof of Theorem 2.1. The most surprising part in the proof is a definition: Gromov assigns to every ball $B_{r}(p) \subset M$ a finite number called the corank of the ball. It is defined as the maximum over all $k$ such that for all $q \in B_{2 r}(p)$ there are points $q_{1}, \ldots, q_{k}$ with

$$
d\left(q, q_{1}\right) \geq 2^{n+3} r, d\left(q, q_{i+1}\right) \geq 2^{n} d\left(p, q_{i}\right)
$$

and $q_{i}$ is a critical point of the distance function of $q$ in the sense of Grove and Shiohama. One can show as follows that the corank of a ball is at most $2 n$ : Choose a minimal geodesic $c_{i j}$ from $q_{i}$ to $q_{j}, i<j$ and minimal geodesic $c_{i}$ from $q$ to $q_{i}, i=1, \ldots, k$. Since $q_{i}$ is a critical point we can find a possibly different minimal geodesic $\tilde{c}_{i}$ from $q$ to $q_{i}$ such that the angle of the triangle $\left(\tilde{c}_{i}, c_{j}, c_{i j}\right)$ based at $q_{i}$ is $\leq \pi / 2$. Therefore $L\left(c_{j}\right)^{2} \leq L\left(c_{i j}\right)^{2}+L\left(c_{i}\right)^{2}$. Applying Toponogov's theorem to the triangle $\left(c_{i}, c_{j}, c_{i j}\right)$ gives that the angle $\varphi_{i j}$ between $c_{i}$ and $c_{j}$ satisfies $\tan \left(\varphi_{i j}\right) \geq 2^{n}$. Thus $\varphi_{i j} \geq \pi / 2-2^{-n}$. The upper bound on $k$ now follows from an Euclidean sphere packing argument in $T_{q} M$.

By reverse induction on the corank, one establishes an estimate for the content of a ball cont $\left(B_{r}(p)\right)$ which is defined as the dimension of the image of $H_{*}\left(B_{r}(p)\right)$ in $H_{*}\left(B_{5 r}(p)\right)$. A ball $B_{r}(p)$ with maximal corank is necessarily contractible in $B_{5 r}(p)$ since for some $q \in B_{2 r}(p)$ the distance function of $q$ has no critical points in $B_{8 r}(q) \backslash\{q\}$. This establishes the induction base. It is immediate from the definition that $\operatorname{corank}\left(B_{\rho}(q)\right) \geq \operatorname{corank}\left(B_{r}(p)\right)$ for all $q \in B_{3 r / 2}(p)$ and all $\rho \leq r / 4$. In the induction step one distinguishes between two cases.

In the first case, one assumes that $\operatorname{corank}\left(B_{\rho}(q)\right)>\operatorname{corank}\left(B_{r}(p)\right)$ for all $q \in B_{r}(p)$ and $\rho:=\frac{r}{4^{n}}$. Using the Bishop Gromov inequality it is easy to find a covering of $B_{r}(q)$ with at most $4^{n(n+2)}$ balls of radius $\rho$. By the induction hypothesis the balls $B_{\rho}(q)$ have a bounded content. Using a rather involved nested covering argument one can give an explicit estimate of the content of $B_{r}(p)$.

In the remaining case there is one point $q \in B_{r}(p)$ such that $\operatorname{corank}\left(B_{\rho}(q)\right)=\operatorname{corank}\left(B_{r}(p)\right)$ with $\rho=\frac{r}{4^{n}}$. Thus for some point $x \in B_{2 \rho}(q)$ there is no critical point of the distance function of $x$ in $B_{8 r}(x) \backslash B_{2^{-n+3} r}(x)$. This implies that one can homotop $B_{r}(p)$ to a subset of $B_{r / 4}(x)$ in $B_{5 r}(p)$. 
From this it is not hard to deduce that $\operatorname{cont}\left(B_{r / 4}(x)\right) \geq \operatorname{cont}\left(B_{r}(p)\right)$. We have seen above $\operatorname{corank}\left(B_{r / 4}(x)\right) \geq \operatorname{corank}\left(B_{r}(p)\right)$. One can now apply the same argument again with $B_{r}(p)$ replaced by $B_{r / 4}(x)$. Since small balls are contractible, the process has to stop after finitely many steps unless possibly $\operatorname{cont}\left(B_{r}(p)\right)=1$.

Fundamental groups. Fundamental groups of nonnegatively curved manifolds are rather well understood. On the other hand, the known results are essentially the same as for compact manifolds with nonnegative Ricci curvature. In fact there is a general belief that the general structure results for fundamental groups should coincide for the two classes. One of the main tools in this context is the splitting theorem of Toponogov, resp. the splitting theorem of Cheeger and Gromoll [1971]. Recall that a line is a normal geodesic $c: \mathbb{R} \rightarrow(M, g)$ satisfying $d(c(t), c(s))=|t-s|$ for all $t, s \in \mathbb{R}$. By Cheeger and Gromoll's splitting theorem complete manifolds of nonnegative Ricci curvature split as products $\mathbb{R} \times M^{\prime}$ provided they contain lines. In the special case of nonnegative sectional curvature, the result is due to Toponogov.

By the work of Cheeger and Gromoll [1971], the splitting theorem implies that a nonnegatively curved manifold $M$ is isometric to $\mathbb{R}^{k} \times B$ where $B$ has a compact isometry group. The same results holds for the universal cover of a compact manifold $M$ of nonnegative Ricci curvature. As a consequence they deduced that the fundamental group of $M$ is virtually abelian, i.e., it contains an abelian subgroup of finite index. Moreover one can show

THEOREM 2.2. Let $(M, g)$ be a compact manifold of nonnegative Ricci curvature or an open manifold of nonnegative sectional curvature. Then there is a family of complete metrics $g_{t}$ on $M$ with $g_{0}=g,\left(M, g_{t}\right)$ is locally isometric to $(M, g)$ for all $t$ and $\left(M, g_{1}\right)$ is finitely covered by a Riemannian product $T^{d} \times M^{\prime}$, where $M^{\prime}$ is simply connected and $T^{d}$ is a flat torus.

The theorem is due to author [2000] but is based on a slightly weaker version of Cheeger and Gromoll [1971]. Moreover, it was shown in [Wilking, 2000] that any finitely generated virtually abelian fundamental group occurs in some dimension as the fundamental group of a nonnegatively curved manifold. However, the more interesting and challenging problem is what one can say about fundamental groups in a fixed dimension.

To the best of the authors knowledge the only other "effective" result known for fundamental groups of nonnegative sectional curvature is

TheOREM 2.3 (Gromov, 1978). The fundamental group of a nonnegatively curved $n$-manifold is generated by at most $n \cdot 2^{n}$ elements.

The proof of the theorem is a simple application of Toponogov's theorem applied to the short generating system of $\pi_{1}(M, p)$. 
Although we mentioned in the introduction that we will report on results which are based on collapsing techniques, we quote, for the sake of completeness, the following recent theorem of Kapovitch, Petrunin and Tuschmann [2005].

THEOREM 2.4. For each $n$ there is a constant $C(n)$ such that the fundamental group of any compact nonnegatively curved n-manifold $(M, g)$ contains a nilpotent subgroup of index at most $C(n)$.

The theorem remains valid for almost nonnegatively curved manifolds and it improves a similar theorem of Fukaya and Yamaguchi from "solvable" to "nilpotent". The proof relies on a compactness result and it remains an open problem whether one can make the bound effective, in other words whether one can give explicit estimates on $C(n)$. It is also remains open whether in case of nonnegative curvature one can improve it from "nilpotent" to "abelian".

Other structure results. By the Gauss-Bonnet formula a compact nonnegatively curved compact surface is given by $\mathbb{R P}^{2}, \mathbb{S}^{2}, T^{2}$ or the Klein bottle. Due to Hamilton [1982] a compact 3-manifold of nonnegative Ricci curvature and finite fundamental group is diffeomorphic to spherical space form, see Theorem 1.13. In dimension 4 a classification remains open. The best result is a theorem in Kleiner's thesis.

THEOREM 2.5 (Kleiner). Let $(M, g)$ be a nonnegatively curved simply connected 4-manifold. If the isometry group is not finite then $M$ is homeomorphic to $\mathbb{S}^{4}, \mathbb{C P}^{2}, \mathbb{S}^{2} \times \mathbb{S}^{2}$ or to a connected sum $\mathbb{C} \mathbb{P}^{2} \# \pm \mathbb{C P}^{2}$.

The Bott conjecture (see last section) would imply that the theorem remains valid if one removes the assumption on the isometry group. It would be interesting to know whether one can improve the conclusion in Theorem 2.5 from homeomorphic to diffeomorphic. Kleiner never published his thesis, but Searle and Yang [1994] reproved his result. We present a slightly shorter proof which has also the advantage that it does not make use of a signature formula of Bott for four manifolds with Killing fields. This in turn implies that part of the proof carries over to simply connected nonnegatively curved 5-manifolds with an isometric 2-torus action. In fact using minor modifications it is not hard to check that the second rational Betti number of such a manifold is bounded above by 1 .

LEMMA 2.6. Let $\rho: \mathrm{S}^{1} \rightarrow \mathrm{O}(4)$ be a representation such that there is no trivial subrepresentation. Consider the induced action of $\mathrm{S}^{1}$ on the standard sphere $\mathbb{S}^{3}$.

a) Any four pairwise different points $p_{1}, \ldots, p_{4} \in B:=\mathbb{S}^{3} / \mathrm{S}^{1}$ satisfy

$$
\sum_{1 \leq i<j \leq 4} d\left(p_{i}, p_{j}\right) \leq 2 \pi .
$$


and equality occurs if and only if $B$ is isometric to the 2-sphere $\mathbb{S}^{2}(1 / 2)$ of constant curvature 4 and $\left\{p_{1}, p_{2}, p_{3}, p_{4}\right\}=\{ \pm p, \pm q\}$.

b) The diameter of $B$ is equal to $\pi / 2$. In fact for $p \in B$ there is most one point $q \in B$ with $d(p, q) \geq \pi / 2$.

Proof. We may assume that $\rho$ is faithful. If the action of $S^{1}$ is given by the Hopf action, then $B:=\mathbb{S}^{3} / \mathbb{S}^{1}$ is the 2-sphere $\mathbb{S}^{2}(1 / 2)$ of constant curvature 4 . Recall that a triangle in $\mathbb{S}^{2}(1 / 2)$ has perimeter $\leq \pi$ and that equality can only occur if two of the points on the boundary triangle have distance $\pi / 2$. Using this for all triangles $\left\{q_{1}, q_{2}, q_{3}\right\} \subset\left\{p_{1}, p_{2}, p_{3}, p_{4}\right\}$, we get the claimed inequality. Equality can only occur if the four points are on a great circle. A more detailed analysis shows that equality implies $\left\{p_{1}, p_{2}, p_{3}, p_{4}\right\}=\{ \pm p, \pm q\}$.

In general it is easy to construct a distance non-increasing homeomorphism

$$
f: \mathbb{S}^{2}(1 / 2) \rightarrow B .
$$

For the proof notice that $B$ admits an isometric action of a circle $\mathrm{T}^{1}$, since the centralizer of $\rho\left(\mathrm{S}^{1}\right)$ in $\mathrm{SO}(4)$ acts isometrically on $B$, the quotient space $B / \mathrm{T}^{1}$ is isometric to the interval $[0, \pi / 2]$. The same holds for the quotient space $\mathbb{S}^{2}(1 / 2) / T^{1}$. It is now easy to see that the orbits of the $\mathbf{T}^{1}$ action on $\mathbb{S}^{2}(1 / 2)$ are at least as long as the corresponding orbits in $B$.

Finally if the action is not given by the Hopf action, then we can not find four different points $p_{1}, p_{2}, p_{3}, p_{4} \in B$ with $d\left(p_{1}, p_{2}\right)=d\left(p_{3}, p_{4}\right)=\pi / 2$. Since $f$ is distance non-increasing part b) follows as well.

Proof of Theorem 2.5. By Freedman's classification of simply connected topological 4-manifolds, it suffices to show that $\chi(M) \leq 4$. Since the Eulercharacteristic of $M$ equals the Eulercharacteristic of the fixed point set of $\mathrm{S}^{1} \subset \operatorname{Iso}(M, g)$, it suffices to estimate the latter.

We now consider the orbit space $A^{3}:=M^{4} / \mathrm{S}^{1}$ as an Alexandrov space. We first want to rule out that $S^{1}$ has more than four isolated fixed points. Suppose $p_{1}, \ldots, p_{5}$ are pairwise different isolated fixed points in $M$.

We can view these points also as points in the orbit space $A$. Choose a fixed minimal normal geodesic $\gamma_{i j}:[0,1] \rightarrow A$ between $p_{i}$ and $p_{j}$ for $i \neq j$. We may assume $\gamma_{i j}$ and $\gamma_{j i}$ are equal up to a change of direction.

We also consider all angles $\alpha_{i j k}$ between $\gamma_{i j}$ and $\gamma_{i k}$ for all pairwise different $i, j$ and $k$. A simple counting argument shows that there are precisely 30 angles. We next prove two different estimates for the sum of these angles.

For any three points in $\left\{p_{1}, p_{2}, p_{3}, p_{4}, p_{5}\right\}$ we get a triangle. The sum of the angles in the triangle is $\geq \pi$, as $X$ is nonnegatively curved in the Alexandrov sense. Therefore the sum of all 30 angles is $\geq 10 \pi$.

On the other hand we can consider for a fixed point $p_{i}$ all 6 angles based at $p_{i}$. The angles are given as the pairwise distances of four distinct points 
in the space of directions $\Sigma_{p_{i}} X$. Since $\Sigma_{p_{i}} X$ is isometric to the quotient of $\mathbb{S}^{3}$ by a $S^{1}$-action, we infer from Lemma 2.6 that the sum of these 6 angles is $\leq 2 \pi$. This proves that the sum of all 30 angles is at most $10 \pi$.

Hence equality must hold everywhere. It follows that the space of directions at $p_{i}$ is given by a sphere of constant curvature 4 . There are precisely 10 angles of size $\pi / 2$ and for each triangle corresponding to three points in $\left\{p_{1}, \ldots, p_{5}\right\}$ the sum of the angles is $\pi$ and hence precisely one angle in such a triangle equals $\pi / 2$. We may assume $d\left(p_{1}, p_{2}\right)=\min _{i \neq j} d\left(p_{i}, p_{j}\right)$. For one point $q \in\left\{p_{3}, p_{4}, p_{5}\right\}$ the triangle $\left(p_{1}, p_{2}, q\right)$ has neither an angle $\pi / 2$ at $p_{1}$ nor an angle $\pi / 2$ at $p_{2}$. Thus there is an angle $\pi / 2$ at $q$. Since equality holds in Toponogov's comparison theorem we see

$$
d\left(p_{1}, q\right)^{2}+d\left(p_{2}, q\right)^{2}=d\left(p_{1}, p_{2}\right)^{2}
$$

a contradiction since $d\left(p_{1}, p_{2}\right)$ was minimal.

Suppose next that the fixed point set $\operatorname{Fix}\left(\mathrm{S}^{1}\right)$ of $\mathrm{S}^{1}$ contains at least two 2-dimensional components. These components form totally geodesic submanifolds of the Alexandrov space $A$. Since they do not intersect it is easy to see that $A$ is isometric to $F \times[0, l]$ where $F$ is a fixed point component. In particular $S^{1}$ has no fixed points outside the two components. Since each component has Eulercharacteristic $\leq 2$ the result follows.

It remains to consider the case that $S^{1}$ has precisely one 2-dimensional fixed point component $F$. We have to show that the $S^{1}$-action has at most two isolated fixed points. Notice that $F$ is the boundary of the Alexandrov space $A$ and the distance function $h:=d(F, \cdot): A \rightarrow \mathbb{R}$ is concave. Let $p \in A$ denote one isolated fixed point with minimal distance $r$ to the boundary. The set $h^{-1}\left(\left[r, \infty[)\right.\right.$ is convex. Let $v \in \Sigma_{p} A$ be the initial direction of a minimal geodesic from $p$ to $F$. The tangent cone $C_{p} h^{-1}([r, \infty[)$ consist of 'vectors' which have an angle $\geq \pi / 2$ with $v$. From Lemma 2.6 we deduce that $C_{p} h^{-1}\left(\left[r, \infty[)\right.\right.$ is at most one dimensional. Thus the convex set $h^{-1}([r, \infty[)$ is either a point or an interval. By construction $h^{-1}([r, \infty[)$ contains all isolated fixed points of $S^{1}$. Since for each fixed point the space of direction has diameter $\pi / 2$, we deduce that there are at most two isolated fixed points.

Gursky and LeBrun [1999] obtained strong restrictions on 4-dimensional nonnegatively curved Einstein manifolds.

One might ask whether any nonnegatively curved compact manifold with finite fundamental group also admits nonnegatively curved metrics with positive Ricci curvature. A partial result in direction was proved recently.

TheOREM 2.7 (Böhm and Wilking, 2005). Let $(M, g)$ be a compact nonnegatively curved manifold with finite fundamental group, and let $g_{t}$ be a solution of the Ricci flow. Then for all small $t>0, g_{t}$ has positive Ricci curvature. 
The proof applies a dynamical version of Hamilton's maximum principle to a family of curvature conditions lying in between nonnegative sectional curvature and nonnegative Ricci curvature. It then follows that $g_{t}$ has nonnegative Ricci curvature for $t \in[0, \varepsilon]$ with $\varepsilon$ depending on an upper curvature bound. Then the theorem follows easily from a strong maximum principle. In the same paper it was also shown that there is no Ricci flow invariant curvature condition in between nonnegative sectional curvature and nonnegative Ricci curvature in dimensions above 11 . This in turn generalized previous results saying that neither nonnegative Ricci curvature nor nonnegative sectional curvature are invariant under the Ricci flow in dimensions above 3 , see $[\mathrm{Ni}, 2004]$.

In particular, any compact nonnegatively curved manifold with finite fundamental group satisfies all obstructions coming from positive Ricci curvature. In the simply connected case the only general known obstruction for positive Ricci curvature is that the manifold admits a metric with positive scalar curvature. By the work of Gromov and Lawson and Stolz the latter statement is equivalent to saying: Either $M$ is not spin or $M$ is a spin manifold with a vanishing $\alpha$-invariant. For more details and references we refer the reader to the surveys of Jonathan Rosenberg and Guofang Wei published in this volume.

Grove-Ziller examples. Recently Grove and Ziller generalized a gluing technique which by the work of Cheeger [1973] was previously only known to work in the special case of connected sums of two rank one symmetric spaces. Since they are discussed in more detail in the survey of Wolfgang Ziller we will be brief.

TheOREM 2.8 (Grove and Ziller, 2000). Let $\mathrm{G}$ be a compact Lie group, and let $\mathrm{G} / / \mathrm{H}$ be a compact biquotient. Suppose there are two subgroups $\mathrm{K}_{ \pm} \subset$ $\mathrm{G} \times \mathrm{G}$ such that $\mathrm{K}_{ \pm} / \mathrm{H} \cong \mathbb{S}^{1}$ and the action of $\mathrm{K}_{ \pm}$on $\mathrm{G}$ is free. Then the manifold obtained by gluing the two disc bundles associated to the two sphere bundles $\mathrm{G} / / \mathrm{H} \rightarrow \mathrm{G} / / \mathrm{K}_{ \pm}$along their common boundary $\mathrm{G} / / \mathrm{H}$ has a metric of nonnegative sectional curvature.

The stated theorem is slightly more general than the original version of Grove and Ziller, who considered cohomogeneity one manifolds or equivalently the case where all groups $\mathrm{H}, \mathrm{K}_{ \pm}$act from the right on $\mathrm{G}$ and hence the corresponding quotients are homogeneous. Of course it would be interesting to know whether the generalization gives rise to any interesting new examples. One can actually reduce the more general statement to the one of Grove and Ziller as follows

Proof. We consider the manifold $M$ which admits a cohomogeneity one action of $\mathrm{G} \times \mathrm{G}$ with principal isotropy group $\mathrm{H}$ and singular isotropy groups $\mathrm{K}_{ \pm} \subset \mathrm{G} \times \mathrm{G}$. By Grove and Ziller this manifold has an invariant metric of nonnegative sectional curvature, see the survey of Wolfgang Ziller for details. By assumption the diagonal $\Delta \mathrm{G} \subset \mathrm{G} \times \mathrm{G}$ acts freely on $M$. Clearly 
the manifold in the theorem is the quotient $M / \Delta \mathrm{G}$. Thus the result follows from the O'Neill formulas.

TheOREM 2.9 (Grove and Ziller). Any principal $\mathrm{SO}(n)$-bundle over $\mathbb{S}^{4}$ admits a cohomogeneity one action of $\mathrm{S}^{3} \times \mathrm{SO}(n)$ with singular orbits of codimension 2 .

The proof uses the classification of bundles over $\mathbb{S}^{4}$ in terms of characteristic classes. Grove and Ziller endow $\mathbb{S}^{4}$ with the unique cohomogeneity one action of $S^{3}$ with singular orbits of codimension 2 . Then they compute for all $\mathrm{S}^{3} \times \mathrm{SO}(n)$-cohomogeneity one manifolds which are $\mathrm{SO}(n)$-principal bundles over the given cohomogeneity one manifold $\mathbb{S}^{4}$ all characteristic classes. By comparing the set of invariants, it follows that one gets all bundles this way. The details are involved.

By taking quotients of such principal bundles it follows that any sphere bundle over $\mathbb{S}^{4}$ admits a metric of nonnegative sectional curvature. This is particular striking since 10 of the 14 exotic spheres in dimension 7 can be realized as such bundles.

Grove and Ziller conjectured in their paper that any cohomogeneity one manifold admits an invariant nonnegatively curved metric. A partial answer was given by Schwachhöfer and Tuschmann [2004] who showed that these manifolds admit metrics of almost nonnegative sectional curvature. However, counterexamples to the Grove-Ziller conjecture were recently found by Grove, Verdiani, Wilking and Ziller [2006]. The counterexamples contain all higher dimensional Kervaire spheres and therefore all exotic spheres of cohomogeneity one. Additional counterexamples are given but to this day it remains an open question how big the class of nonnegatively curved cohomogeneity one manifolds is.

\section{Open nonnegatively curved manifolds}

Noncompact nonnegatively curved spaces often occur as blow up limits of sequences of manifolds converging with lower curvature bound -1 to a limit. Also recall a result of Hamilton and Ivey saying that for any singularity of the Ricci flow in dimension 3 the corresponding blow up limit has nonnegative sectional curvature. This in turn was one key feature which allowed Hamilton and Perelman to classify the possible singularities of the Ricci flow in dimension 3.

By a result of Gromov [1986] any noncompact manifold admits a positively curved metric. However Gromov's metrics are not complete and we assume throughout the paper that all metrics are complete.

The structure of open manifolds of nonnegative (positive) sectional curvature is better understood than the compact case. By a theorem of Gromoll and Meyer [1969] a positively curved open manifold is diffeomorphic to the Euclidean space. For a nonnegatively curved manifold there is the soul theorem. 
Theorem 3.1 (Cheeger and Gromoll, 1971). For an open nonnegatively curved manifold $M$ there is a totally geodesic submanifold $\Sigma$ called the soul such that $M$ is diffeomorphic to the normal bundle of $\Sigma$.

Sketch OF THE PROOF. The basic observation in the proof is that for each point $p \in M$ the function $f_{0}(q):=\lim _{r \rightarrow \infty} d\left(\partial B_{r}(p), q\right)-r$ is concave, proper and bounded above. Hence the maximal level of $f_{0}$ is a convex closed subset $C_{1}$ of $M$. Cheeger and Gromoll showed that $C_{1}$ is a totally geodesic compact submanifold with a possibly non-empty and non-smooth intrinsic boundary $\partial C_{1}$. One can then show that if $\partial C_{1} \neq \emptyset$, then the function $f_{1}(q)=$ $d\left(\partial C_{1}, q\right)$ is concave on $C_{1}$. As before the maximal level set $C_{2}$ of $f_{1}$ is a convex subset of $M$. Since $\operatorname{dim}\left(C_{2}\right)<\operatorname{dim}\left(C_{1}\right)$ one can iterate the process until one arrives at a convex level set $C_{k}$ without intrinsic boundary. Then $\Sigma:=C_{k}$ is a soul of $M$. One can show that the distance function $r_{\Sigma}:=d(\Sigma, \cdot)$ has no critical points on $M \backslash \Sigma$ in the sense of Grove and Shiohama, for a definition see section 1 . Thus there is a gradient like vectorfield $X$ on $M \backslash \Sigma$, with $\|X\| \leq 1$. Similarly to the proof of the diameter sphere theorem one can now construct a diffeomorphism $\psi: \nu(\Sigma) \rightarrow M$.

We emphasize that the diffeomorphism $\nu(\Sigma) \rightarrow M$ is in general not given by the exponential map. On the other hand it was shown by Guijarro [1998], that there is always at least one complete nonnegatively curved metric on $M$ such that this is the case.

From the soul construction it is clear that there is a Hausdorff continuous family $(C(s))_{s \in[0, \infty)}$ of convex compact subsets of $M$ such that $C(0)=\Sigma$, $C\left(s_{1}\right) \subset C\left(s_{2}\right)$ for $s_{1}<s_{2}$ and $\bigcup_{s>0} C(s)=M$. In fact from the above sketch this family can be obtained by collecting all nonempty sublevels $f_{i}^{-1}\left(\left[c, \infty[)\right.\right.$ of the functions $f_{0}, \ldots, f_{k-1}$ in one family. Given such a family, Sharafutdinov [1979] showed, independent of curvature assumptions, that there is a distance non-increasing retraction $P: M \rightarrow \Sigma$.

Theorem 3.2 (Perelman, 1994). Let $\Sigma$ be a soul of $M, \nu(\Sigma)$ its normal bundle and $P: M \rightarrow \Sigma$ a Sharafutdinov retraction. Then

a) $P \circ \exp _{\nu(\Sigma)}=\pi$, where $\pi: \nu(\Sigma) \rightarrow \Sigma$ denotes the projection.

b) Each two vectors $u \in \nu_{p}(\Sigma)$ and $v \in T_{p} \Sigma$ are tangent to a totally geodesic immersed flat $\mathbb{R}^{2}$.

c) $P$ is a Riemannian submersion of class $C^{1}$.

The theorem also confirmed the soul conjecture of Cheeger and Gromoll: A nonnegatively curved open manifold with positive sectional curvature at one point is diffeomorphic to $\mathbb{R}^{n}$. Although this conjecture was open for more than two decades, the proof of the above theorem is very short and just uses Rauch's comparison theorem.

Guijarro [2000] showed that $P$ is of class $C^{2}$ and it was shown in [Wilking, $2005]$ that $P$ is of class $C^{\infty}$. The latter result is a consequence of another 
structure theorem on open nonnegatively curved manifolds whose explanation requires a bit of preparation: One defines a dual foliation $\mathcal{F}^{\#}$ to the foliation $\mathcal{F}$ given by the fiber decomposition $P: M \rightarrow \Sigma$ as follows. For a point $p \in M$ we define the dual leaf $\mathcal{L}^{\#}(p)$ as the set of all points which can be connected with $p$ by a piecewise horizontal curve. We recall that a curve is called horizontal with respect to $P$, if it is everywhere perpendicular to the fibers of $P$.

Because of Theorem 3.2 each dual leaf can also be obtained as follows. Consider a vector $v$ in the normal bundle $\nu(\Sigma)$ of the soul. Let $S(v)$ denote set of all vectors in $\nu(\Sigma)$ which are parallel to $v$ along some curve in $\Sigma$. Then $\exp (S(v))=\mathcal{L}^{\#}(\exp (v))$. The structure of the dual foliation is thus closely linked to the normal holonomy group of the soul.

If the normal holonomy group is transitive on the sphere, then the dual leaves are just given by distance spheres to the soul. If the holonomy group is trivial, then by a result of Strake [1988] and Yim [1990] $M^{n}$ splits isometrically as $\Sigma^{k} \times\left(\mathbb{R}^{n-k}, g\right)$ and the dual leaves are just given by isometric copies of $\Sigma$. In general the holonomy group is neither transitive nor trivial. In fact, by an unpublished result of the author, any connected subgroup of $\mathrm{SO}(n-k)$ can occur as the normal holonomy group of a simply connected soul.

TheOREM 3.3 (Wilking, 2005). Let $M, \Sigma, P, \mathcal{F}^{\#}$ be as above.

a) Then $\mathcal{F}^{\#}$ is a singular Riemannian foliation, i.e., geodesics emanating perpendicularly to dual leaves stay perpendicularly to dual leaves.

b) If $u \in T_{p} M$ is horizontal with respect to $P$ and $v \in T_{p} M$ is perpendicular to the dual leaf $\mathcal{L}^{\#}(p)$, then $u$ and $v$ are tangent to a totally geodesic immersed flat $\mathbb{R}^{2}$.

An analogous theorem holds for Riemannian submersions on compact nonnegatively curved manifolds. A consequence of the above theorem is that any non-contractible open nonnegatively curved manifold has an honest product as a metric quotient.

Corollary 3.4. Let $M$ be an open nonnegatively curved manifold and $\Sigma$ a soul of $M$. Then there is a noncompact Alexandrov space $A$ and a submetry

$$
\sigma: M \rightarrow \Sigma \times A
$$

onto the metric product $\Sigma \times A$. The fibers of $\sigma$ are smooth compact submanifolds without boundary.

We recall that $\sigma: M \rightarrow B$ is called a submetry if $\sigma\left(B_{r}(p)\right)=B_{r}(\sigma(p))$ for all $p$ and $r$. The space $A$ is given by the space of closures of dual leaves, which by Theorem 3.3 can be endowed with a natural quotient metric.

The main new tool used to prove these results is a simple and general observation which may very well be useful in different context as well. 
It allows to give what we call transversal Jacobi field estimates. Let $c: I \rightarrow$ $(M, g)$ be a geodesic in a Riemannian manifold $(M, g)$, and let $\Lambda$ be an $(n-1)$-dimensional family of normal Jacobi fields for which the corresponding Riccati operator is self adjoint. Recall that the Riccati operator $L(t)$ is the endomorphism of $(\dot{c}(t))^{\perp}$ defined by $L(t) J(t)=J^{\prime}(t)$ for $J \in \Lambda$. Suppose we have a vector subspace $\Upsilon \subset \Lambda$. Put

$$
T_{c(t)}^{v} M:=\{J(t) \mid J \in \Upsilon\} \oplus\left\{J^{\prime}(t) \mid J \in \Upsilon, J(t)=0\right\} .
$$

Observe that the second summand vanishes for almost every $t$ and that $T_{c(t)}^{v} M$ depends smoothly on $t$. We let $T_{c(t)}^{\perp} M$ denote the orthogonal complement of $T_{c(t)}^{v} M$, and for $v \in T_{c(t)} M$ we define $v^{\perp}$ as the orthogonal projection of $v$ to $T_{c(t)}^{\perp} M$. If $L$ is non-singular at $t$ we put

$$
A_{t}: T_{c(t)}^{v} M \rightarrow T_{c(t)}^{\perp} M, J(t) \mapsto J^{\prime}(t)^{\perp} \text { for } J \in \Upsilon .
$$

It is easy to see that $A$ can be extended continuously on $I$. For a vector field $X(t) \in T_{c(t)}^{\perp} M$ we define $\frac{\nabla^{\perp} X}{\partial t}=\left(X^{\prime}(t)\right)^{\perp}$.

TheOREM 3.5. Let $J \in \Lambda-\Upsilon$ and put $Y(t):=J^{\perp}(t)$. Then $Y$ satisfies the following Jacobi equation

$$
\frac{\left(\nabla^{\perp}\right)^{2}}{\partial t^{2}} Y(t)+(R(Y(t), \dot{c}(t)) \dot{c}(t))^{\perp}+3 A_{t} A_{t}^{*} Y(t)=0
$$

One should consider $(R(\cdot, \dot{c}(t)) \dot{c}(t))^{\perp}+3 A_{t} A_{t}^{*}$ as the modified curvature operator. The crucial point in the equation is that the additional O'Neill type term $3 A_{t} A_{t}^{*}$ is positive semidefinite.

Corollary 3.6. Consider an $n$-1-dimensional family $\Lambda$ of normal Jacobi fields with a self adjoint Riccati operator along a geodesic $c: \mathbb{R} \rightarrow M$ in a nonnegatively curved manifold. Then

$$
\Lambda=\operatorname{span}_{\mathbb{R}}\{J \in \Lambda \mid J(t)=0 \text { for some } t\} \oplus\{J \in \Lambda \mid J \text { is parallel }\} .
$$

3.1. Which bundles occur? The major open problem in the subject is

Problem 1. Let $(\Sigma, g)$ be a nonnegatively curved compact manifold. Which vectorbundles $E$ over $\Sigma$ admit nonnegatively curved metrics such that the zero section of the bundle is a soul?

If $L$ is a nonnegatively curved compact manifold with a free isometric $\mathrm{O}(k)$ action, then the corresponding bundle $L \times{ }_{\mathrm{O}(k)} \mathbb{R}^{k}$ has a nonnegatively curved metric with the zero section being the soul. It is remarkable that all examples of open nonnegatively curved manifolds constructed so far are diffeomorphic to examples arising in this way. On the other hand the above method is rather flexible already. From Theorem 2.8 it follows. 
ThEOREM 3.7 (Grove and Ziller). All vectorbundles over $\mathbb{S}^{4}$ admit complete nonnegatively curved metrics.

It is not known whether one can find nonnegatively metrics such that the souls are isometric to the round sphere. The souls of the Grove-Ziller metrics have lots zero curvature planes. All of the relatively few vectorbundles over $\mathbb{S}^{5}$ also admit nonnegatively curved metrics [Rigas, 1985]. However, in general Cheeger and Gromoll's question which bundles over a sphere admit nonnegatively curved metrics remains open.

We mention in some cases one can say a bit more about which bundles occur: if either the soul has infinite fundamental group or if one fixes the isometry type of the soul. Özaydin and Walschap [1994] observed that a flat soul necessarily has a flat normal bundle. If one has an open manifold with infinite fundamental group then, by Theorem 2.2 one can deform the metric within the space of nonnegatively curved metrics such that a finite cover is isometric to $T^{d} \times M$, where $M$ is simply connected. This in turn shows that the normal bundle of the soul $T^{d} \times \Sigma^{\prime}$ is canonically isomorphic to the pull back of a bundle over the simply connected factor $\Sigma^{\prime}$. The question whether such a bundle can also be written as a twisted bundle over $T^{d} \times \Sigma^{\prime}$ was studied in great detail by Belegradek and Kapovitch [2003] using rational homotopy theory.

Moreover one can analyze the situation if the soul is isometric to a simply connected product $\Sigma=\Sigma_{1} \times \Sigma_{2}$. Although this is just an observation due to the author we carry out some details here since they can not be found in the literature. If $u_{i} \in T_{p} \Sigma$ is tangent to the $i$-th factor $(i=1,2)$, then $R\left(u_{1}, u_{2}\right) v=0$ for $v \in \nu_{p}(\sigma)$. By "integrating" this equation we deduce that for a closed curve $\gamma(t)=\left(\gamma_{1}(t), \gamma_{2}(t)\right)$ the normal parallel transport $\operatorname{Par}_{\gamma}$ decomposes $\operatorname{Par}_{\gamma}=\operatorname{Par}_{\gamma_{1}} \circ \operatorname{Par}_{\gamma_{2}}=\operatorname{Par}_{\gamma_{2}} \circ \operatorname{Par}_{\gamma_{1}}$. Thus the normal holonomy group is given as the product of two commuting subgroups. Each subgroup gives rise to a principle bundle over $\Sigma$ which is isomorphic to the pull back bundle of a principle bundle over $\Sigma_{i}$ under the natural projection $\Sigma \rightarrow \Sigma_{i}$. If we decompose the normal bundle into parallel subbundles $\nu(\Sigma)=\nu_{1}(\Sigma) \oplus \cdots \oplus \nu_{l}(\Sigma)$ such that on each summand the holonomy group is irreducible, then each summand is isomorphic to a tensor product $\nu_{i}(\Sigma)=\nu_{i 1}(\Sigma) \otimes_{\mathbb{K}} \nu_{i 2}(\Sigma)$ where $\nu_{i j}(\Sigma)$ is isomorphic to the pull back of a $\mathbb{K}$ vectorbundle bundle over $\Sigma_{j}$ under the natural projection $\Sigma \rightarrow \Sigma_{j}, j=1,2$ and $\mathbb{K} \in\{\mathbb{R}, \mathbb{C}, \mathbb{H}\}$ depends on $i$.

Since any vectorbundle over $\mathbb{S}^{3}$ is trivial, we deduce.

Corollary 3.8. Suppose the soul is isometric to a product $\mathbb{S}^{3} \times \mathbb{S}^{3}$ then the normal bundle of the soul is trivial.

3.2. The space of nonnegatively curved metrics. Perelman's theorem indicates that the moduli space of metrics should be rather small. On the other hand one can not expect too much. Belegradek used the method of Grove and Ziller to exhibit the following phenomena. 
THEOREM 3.9 (Belegradek). There is a non-compact manifold $M$ that admits a sequence of complete nonnegatively curved metrics $\left(g_{k}\right)_{k \in \mathbb{N}}$ such that the souls of $\left(M, g_{k}\right)$ are pairwise non-diffeomorphic.

The theorem shows that the moduli space of nonnegatively curved metrics on $M$ has infinitely many components. This is in sharp contrast to the space of nonnegatively curved metrics on $\mathbb{S}^{2} \times \mathbb{R}^{2}$.

THEOREM 3.10 (Gromoll and Tapp). Up to a diffeomorphism a nonnegatively curved metric on $\mathbb{S}^{2} \times \mathbb{R}^{2}$ is either a product metric or the metric is invariant under the effective action of a two torus and it can be obtained as a quotient of a product metric on $\mathbb{S}^{2} \times \mathbb{R}^{2} \times \mathbb{R}$ by a free $\mathbb{R}$-action.

For a nontrivial 2-dimensional vector bundles over $\mathbb{S}^{2}$ the space of nonnegatively curved metrics is somewhat more flexible. In fact Walschap [1988] showed that given an open four manifold with a soul $\mathbb{S}^{2}$ for which any zero curvature plane is tangent to one of the Perelman flats from Theorem 3.2 the following holds: Let $\frac{\partial}{\partial \varphi}$ denote one of the two unit vectorfields in $M \backslash \Sigma$ tangent to the fibers of the Sharafutdinov retraction and whose integral curves have constant distance to the soul. If $f$ is an arbitrary function on $M$ with compact support contained in $M \backslash \Sigma$, then the following metric has nonnegative sectional curvature as well,

$$
g_{t}(u, v):=g(u, v)+t f(p) g\left(u, \frac{\partial}{\partial \varphi}\right) g\left(v, \frac{\partial}{\partial \varphi}\right)
$$

for all $u, v \in T_{p} M$ and all small $t$.

A partial rigidity result was established by Guijarro and Petersen [1997],

TheOREM 3.11. Let $(M, g)$ be an open nonnegatively curved manifold and $p \in M$. Suppose that for any sequence $p_{n} \in M$ converging to $\infty$ the corresponding sequence $\operatorname{scal}\left(p_{n}\right)$ of scalar curvatures tends to 0 . Then the soul of $M$ is flat.

\section{Positively curved manifolds with symmetry}

Grove (1991) suggested to classify manifolds of positive sectional curvature with a large isometry group. The charm of this proposal is that everyone who starts to work on this problem is himself in charge of what 'large' means. One can relax the assumption if one gets new ideas. One potential hope could be that if one understands the obstructions for positively manifolds with a 'large' amount of symmetry, one may get an idea for a general obstruction. However the main hope of Grove's program is that the process of relaxing the assumptions should lead toward constructing new examples.

That this can be successful was demonstrated by the classification of simply connected homogeneous spaces of positive sectional curvature carried out by Berger [1961], Wallach [1972], Aloff Wallach [1975] and Berard 
Bergery [1976]. The classification led to new examples in dimension 6, 7 and 12, 13 and 24. For the sake of completeness it should be said that the only other source of known positively curved examples are biquotients, i.e., quotients $\mathrm{G} / / \mathrm{H}$, where $\mathrm{G}$ is a compact Lie group and $\mathrm{H}$ is a subgroup of $\mathrm{G} \times \mathrm{G}$ acting freely on $\mathrm{G}$ from the left and the right. Eschenburg [1982] and Bazaikin [1996] found infinite series of such examples in dimensions 7 and 13. We refer the reader to the survey of Wolfgang Ziller for more details.

Another motivation for Grove's proposal was the following theorem.

Theorem 4.1 (Hsiang and Kleiner, 1989). Let $M^{4}$ be an orientable compact 4-manifold of positive sectional curvature. Suppose that there is an isometric nontrivial action of $\mathrm{S}^{1}$ on $M^{4}$. Then $M^{4}$ is homeomorphic to $\mathbb{S}^{4}$ or $\mathbb{C} \mathbb{P}^{2}$.

The theorem is a special case of Theorem 2.5. Grove and Searle [1994] realized that the proof of the above theorem can be phrased naturally in terms of Alexandrov geometry of the orbit space $M^{4} / \mathrm{S}^{1}$. A careful analysis of the orbit space also allowed them to establish the following result.

Theorem 4.2 (Grove and Searle). Let $M^{n}$ be an orientable compact Riemannian manifold of positive sectional curvature. Then

$$
\operatorname{symrank}(M, g):=\operatorname{rank}(\operatorname{Iso}(M, g)) \leq\left[\frac{n+1}{2}\right]
$$

and if equality holds, then $M$ is diffeomorphic to $\mathbb{S}^{n}, \mathbb{C P}^{n / 2}$ or to a lens space.

The inequality is a simple consequence of a theorem of Berger saying that a Killing field on an even dimensional positively curved manifold has a zero. For the equality discussion Grove and Searle first show, that there in an isometric $\mathrm{S}^{1}$ action on $M$ such that the fixed point set has a component $N$ of codimension 2. They then prove that the distance function $d(N, \cdot)$ has no critical points in $M \backslash N$ except for precisely one $\mathrm{S}^{1}$-orbit where it attains its maximum. This is used to recover the structure of the manifold.

Another result which essentially relies on the study of the orbit space is due to Rong [2002]. He showed that a simply connected positively curved 5 -manifold with symmetry rank 2 is diffeomorphic to $\mathbb{S}^{5}$.

Recently, the author made the following basic observation, see [Wilking, 2003].

Theorem 4.3 (Connectedness Lemma). Let $M^{n}$ be a compact Riemannian manifold with positive sectional curvature.

a) Suppose $N^{n-k} \subset M^{n}$ is a compact totally geodesic embedded submanifold. Then the inclusion map $N^{n-k} \rightarrow M^{n}$ is $n-2 k+1$ connected. If there is a Lie group $\mathrm{G}$ that acts isometrically on $M^{n}$ and fixes $N^{n-k}$ pointwise, then the inclusion map is $n-2 k+1+\delta(\mathrm{G})$ connected where $\delta(\mathrm{G})$ is the dimension of the principal orbit. 
b) Suppose $N_{1}^{n-k_{1}}, N_{2}^{n-k_{2}} \subset M^{n}$ are two compact totally geodesic embedded submanifolds, $k_{1} \leq k_{2}, k_{1}+k_{2} \leq n$. Then the intersection $N_{1}^{n-k_{1}} \cap N_{2}^{n-k_{2}}$ is a totally geodesic embedded submanifold as well and the inclusion

$$
N_{1}^{n-k_{1}} \cap N_{2}^{n-k_{2}} \rightarrow N_{2}^{n-k_{2}}
$$

is $n-k_{1}-k_{2}$ connected.

Theorem 4.3 turns out to be a very powerful tool in the analysis of group actions on positively curved manifolds. In fact by combining the theorem with the following lemma, one sees that a totally geodesic submanifold of low codimension in a positively curved manifold has immediate consequences for the cohomology ring of the manifold.

Lemma 4.4. Let $M^{n}$ be a closed differentiable oriented manifold, and let $N^{n-k}$ be an embedded compact oriented submanifold without boundary. Suppose the inclusion $N^{n-k} \rightarrow M^{n}$ is $n-k-l$ connected and $n-k-2 l>0$. Let $[N] \in H_{n-k}(M, \mathbb{Z})$ be the image of the fundamental class of $N$ in $H_{*}(M, \mathbb{Z})$ and let $e \in H^{k}(M, \mathbb{Z})$ be its Poincare dual. Then the homomorphism

$$
\cup e: H^{i}(M, \mathbb{Z}) \rightarrow H^{i+k}(M, \mathbb{Z})
$$

is surjective for $l \leq i<n-k-l$ and injective for $l<i \leq n-k-l$.

Notice that in the case of a simply connected manifold $M$ the submanifold $N$ is simply connected as well and hence it is orientable. Recall that the pull back of $e$ to $H^{k}(N, \mathbb{Z})$ is the Euler class of the normal bundle of $N$ in $M$.

Part b) of the Theorem 4.3 says in particular that $N_{1}^{n-k_{1}} \cap N_{2}^{n-k_{2}}$ is not empty which is exactly the content of Frankel's Theorem. In fact similarly to Frankel's Theorem a Synge type argument is crucial in the proof of Theorem 4.3. The proof of Theorem 4.3 is a very simple Morse theory argument in the space of all curves from $N$ to $N$, respectively from $N_{1}$ to $N_{2}$. The critical points of the energy functional are geodesics starting and emanating perpendicularly to the submanifolds. Using the second variation formulas it is then easy to give lower bounds on the indices of the nontrivial critical points.

The above result is the main new tool that is used in [Wilking, 2003] to show.

THEOREM 4.5. Let $M^{n}$ be a simply connected $n$-dimensional manifold of positive sectional curvature, $n \geq 8$, and let $d \geq \frac{n}{4}+1$. Suppose that there is an effective isometric action of a torus $\mathrm{T}^{d}$ on $M^{n}$. Then $M$ is homotopically equivalent to $\mathbb{C P}^{n / 2}$ or homeomorphic to $\mathbb{H} \mathbb{P}^{n / 4}$ or $\mathbb{S}^{n}$.

In dimensions 8 and 9 the theorem is due to Fang and Rong [2005]. Thus dimensions 6, 7 remain the only dimensions where one needs maximal symmetry rank assumptions for a classification. 
If $M^{n}$ is an odd-dimensional manifold, that is not simply connected but satisfies all other assumptions of the theorem, then its fundamental group is cyclic, see Rong [2000]. A conjecture of Mann [1965] asserts that an exotic sphere $\Sigma^{n}$ can not support an effective smooth action of a $d$-dimensional torus with $d \geq \frac{n}{4}+1$.

Notice that $\mathrm{F}_{4}$, the isometry group of $\mathrm{CaP}^{2}$ has rank 4 . Thus in dimension 16 the result is optimal. Similarly the isometry group of the 12-dimensional Wallach flag has rank 3. In dimension 13 the Berger space $\mathrm{SU}(5) / \mathrm{S}^{1} \cdot \mathrm{Sp}(2)$ is an optimal counterexample.

There are three major constants to measure the amount of symmetry of a Riemannian manifold $(M, g)$ :

$$
\begin{gathered}
\operatorname{symrank}(M, g)=\operatorname{rank}(\operatorname{Iso}(M, g)), \\
\operatorname{symdeg}(M, g)=\operatorname{dim}(\operatorname{Iso}(M, g)) \\
\operatorname{cohom}(M, g)=\operatorname{dim}((M, g) / \operatorname{Iso}(M, g)) .
\end{gathered}
$$

So far we have mostly considered the first of these constants.

TheOREM 4.6 (Wilking, 2006). Let $\left(M^{n}, g\right)$ be a simply connected Riemannian manifold of positive sectional curvature. If $\operatorname{symdeg}\left(M^{n}, g\right) \geq$ $2 n-6$, then $(M, g)$ is tangentially homotopically equivalent to a rank 1 symmetric space or isometric to a homogeneous space of positive sectional curvature.

Notice that all homogeneous spaces of positive sectional curvature satisfy the assumptions of the theorem. In dimension 7 the theorem gives the optimal bound as there are positively curved Eschenburg space $\mathrm{SU}(3) / / \mathrm{S}^{1}$ with a seven dimensional isometry group.

Finally we consider the cohomogeneity of a Riemannian manifold.

THEOREM 4.7 (Wilking, 2006). Let $k$ be a positive integer. In dimensions above $18(k+1)^{2}$ each simply connected Riemannian manifold $M^{n}$ of cohomogeneity $k \geq 1$ with positive sectional curvature is tangentially homotopically equivalent to a rank one symmetric space.

The proof of Theorem 4.7 actually establishes the existence of an infinite sequence of (connected) Riemannian manifolds

$$
M=M_{0} \subset M_{1} \subset \cdots
$$

such that $\operatorname{dim}\left(M_{i}\right)=n+i h$, where $h \leq 4 k+4$ is a positive integer that depends on $M$. All inclusions are totally geodesic, all manifolds are of cohomogeneity $k$ and all have positive sectional curvature. One then considers $M_{\infty}:=\bigcup M_{i}$. On the one hand one can use the connectedness lemma to show that $M_{\infty}$ has $h$-periodic integral cohomology ring. On the other hand, using Alexandrov geometry of the orbit space, one can show that $M_{\infty}$, has 
the homotopy type of the classifying space of a compact Lie group. The results combined show that $M_{\infty}$ is either contractible or has the homotopy type of $\mathbb{C} \mathbb{P}^{\infty}$ or $\mathbb{H} \mathbb{P}^{\infty}$. The connectedness lemma then implies that $M$ has the corresponding homotopy type. The details are quite involved and we refer the reader to [Wilking, 2006].

Of course one might hope that for small $k$ one can use similar techniques to get a classification in all dimensions, or at least a classification up to some potential candidates for positively curved manifolds.

The following theorem carries out such a program in the case of $k=1$.

Theorem 4.8 (Verdiani, Grove, Wilking, Ziller). Let $M^{n}$ be a simply connected compact Riemannian manifold of positive sectional curvature. Suppose that a connected Lie group G acts by isometries with cohomogeneity one, i.e., the orbit space $M^{n} / \mathrm{G}$ is one dimensional. Then one of the following holds:

- $M^{n}$ is equivariantly diffeomorphic to one of the known positively curved biquotients endowed with a natural cohomogeneity action.

- $n=7$ and $M$ is the two fold cover of a 3-Sasakian manifold that corresponds to one of the self dual Einstein 4-orbifolds of cohomogeneity one that were found by Hitchin.

- $n=7$ and $M$ is equivariantly diffeomorphic to one particular cohomogeneity one manifold.

In even dimensions the theorem is due to Verdiani [2001], in this case only rank 1 symmetric spaces occur. The odd dimensional case is more involved and is due to Grove, Wilking and Ziller [2006]. This is partly due to the fact that in dimensions 7 and 13, there are infinitely many positively curved biquotients of cohomogeneity one.

It remains open whether the last two cases can indeed occur. The proof of the theorem uses a lot of the techniques that we have mentioned above. We refer to the survey of Ziller for a more detailed discussion. Very different results on positively curved manifolds with symmetry were found by Dessai [2005].

TheOREM 4.9. Suppose $(M, g)$ is a positively curved spin manifold of dimension $\geq 12$. Let $\mathrm{G}$ be a connected Lie group acting smoothly and suppose a subgroup $\mathbb{Z}_{2}^{2} \subset \mathrm{G}$ acts by isometries. Then the characteristic number $\hat{A}(M, T M)$ vanishes.

The proof is a clever combination of Frankel's theorem on the intersection of totally geodesic submanifolds and a vanishing theorem of Hirzebruch and Slodowy. The non-vanishing of $\hat{A}(M, T M)$ would by that result ensure that each of the three involutions in $\mathbb{Z}_{2}^{2}$ has a fixed point set of codimension 4 . By Frankel these three components have a common intersection and the contradiction arises by inspecting the isotropy representation of $\mathbb{Z}_{2}^{2}$ at a fixed point. 
In the presence of stronger symmetry assumptions he can show the vanishing of more characteristic numbers. These numbers occur naturally as coefficients of a power series expanding the elliptic genus.

4.1. Manifolds with positive sectional curvature almost everywhere. As mentioned before there are relatively few known examples of positively curved manifolds. The lists of examples is quite bit longer if one includes manifolds which have positive sectional curvature on an open dense set.

The most interesting example in the class is the Gromoll Meyer sphere. Gromoll and Meyer [1974] considered the subgroup $\mathrm{H} \subset \mathrm{Sp}(2) \times \mathrm{Sp}(2)$ given by

$$
\mathrm{H}:=\left\{(\operatorname{diag}(1, q), \operatorname{diag}(q, q)) \mid q \in \mathrm{S}^{3}\right\}
$$

and the induced free two sided action of $\mathrm{H}$ on $\mathrm{Sp}(2)$. They showed that the corresponding biquotient $\Sigma^{7}:=\mathrm{Sp}(2) / / \mathrm{H}$ is diffeomorphic to an exotic sphere.

Furthermore, by the O'Neill formulas the metric on $\mathrm{Sp}(2) / / \mathrm{H}$ induced by the biinvariant metric $g$ on $\mathrm{Sp}(2)$ has nonnegative sectional curvature. In fact it is not hard to see that there is a point $p \in \Sigma^{7}$ such that all planes based at $p$ have positive curvature.

It was shown later by Wilhelm [1996] that there is a left invariant metric on $\operatorname{Sp}(2)$ such that the induced metric on $\Sigma^{7}$ has positive sectional curvature on an open dense set of points. Gromoll and Meyer mention in their paper the so called deformation conjecture:

Problem 2. (Deformation conjecture) Let $M$ be a complete nonnegatively curved manifold for which there is point $p \in M$ such that all planes based at $p$ have positive sectional curvature. Does $(M, g)$ admit a positively curved metric, as well?

In the case of an open manifold $M$ the conjecture is by Perelman's solution of the soul conjecture valid. However in general counter examples were found in [Wilking, 2002].

THEOREM 4.10. The projective tangent bundles $P_{\mathbb{R}} T \mathbb{R} \mathbb{P}^{n}, P_{\mathbb{C}} T \mathbb{C P}^{n}$ and $P_{\mathbb{H}} T H \mathbb{P} \mathbb{P}^{n}$ of the projective spaces admit metrics with positive sectional curvature on open dense sets.

It is easy to see that the projective tangent bundle of $P_{\mathbb{R}} T \mathbb{R} \mathbb{P}^{2 n+1}$ is odd dimensional and not orientable. By a theorem of Synge it can not admit a metric with positive sectional curvature. In dimensions $4 n-1,(n \geq 3)$ there are infinitely many homotopy types of simply connected compact manifolds with positive sectional curvature on open dense sets. In fact one 'half' of the circle bundles over $P_{\mathbb{C}} T \mathbb{C P}^{n}$ give rise to such examples.

It is also interesting to note that the natural inclusions among these examples remain totally geodesic embeddings and that the isometry groups 
of the manifolds act with cohomogeneity 2. By the results on positively manifolds with symmetry, these properties could not persist for positively curved metrics. Another consequence is that $\mathbb{S}^{2} \times \mathbb{S}^{3}$, the universal cover of $P_{\mathbb{R}} T \mathbb{R} \mathbb{P}^{3}$, admits a metric with positive sectional curvature on an open dense set.

Finally we should mention that prior to [Wilking, 2002], Petersen and Wilhelm [1999] constructed a slightly different metric on the unit tangent bundle of $\mathbb{S}^{4}$, the universal cover of $P_{\mathbb{R}} T \mathbb{R} \mathbb{P}^{4}$, with positive curvature on an open dense set.

\section{Open Problems}

In this final section we mention some of the major open problems in the subject. The authors favorite conjecture in this context is the so called Bott-conjecture which was promoted by Grove and Halperin.

ConjeCture 5.1. Any nonnegatively curved manifold is rationally elliptic.

We recall that a manifold is called rationally elliptic if $\pi_{*}(M) \otimes \mathbb{Q}$ is finite dimensional. The conjecture would for example show that the total rational Betti number of a nonnegatively curved manifold $M$ is bounded above by $2^{n}$ with equality if and only if $M$ is a flat torus.

There is a conceptual reason why the Bott-conjecture holds for all known nonnegatively curved manifolds. Up to deformation of metrics all known nonnegatively manifolds are constructed from Lie groups endowed with biinvariant metrics using the following three techniques

- One can take products of nonnegatively curved manifolds.

- One can pass from a nonnegatively curved manifold endowed with a free isometric group action to the orbit space endowed with its submersion metric.

- Due to work of Cheeger[1973] and Grove and Ziller [2000] one can sometimes glue disc bundles, i.e., if $M$ is a nonnegatively curved manifold which is in two ways the total space of a sphere bundle (with the structure group being a Lie group), then sometimes the manifold obtained by glueing the two corresponding disc bundles has nonnegative curvature as well.

It is well known that Lie groups are rationally elliptic. Furthermore, by the exact homotopy sequence the class of rationally elliptic manifolds is invariant under taking quotients of free actions. By the work of Grove and Halperin [1987], a manifold obtained by gluing two disc bundles along their common boundary is rationally elliptic if and only if the boundary is.

Grove suggested that the conjecture should hold more generally for the class of simply connected almost nonnegatively curved manifolds. Here we call a manifold almost nonnegatively curved if it admits a sequence $g_{k}$ of 
metrics with diameter 1 and sectional curvature $\geq-\varepsilon(k) \rightarrow 0$. The latter class contains more known examples. On the other hand the only additional technique needed to construct all of the known simply connected almost nonnegatively curved manifolds is:

- If $M$ is an almost nonnegatively curved manifold and $P$ is a principal G-bundle over $M$ with $\mathrm{G}$ being a compact Lie group, then $P$ has almost nonnegative sectional curvature as well.

Clearly with this method one can not leave the class of rationally elliptic manifolds either. Grove suggested that it might be possible to prove the Bott conjecture by induction on the dimension. In this context he posed the problem whether any compact nonnegatively curved manifold has a nontrivial collapse: Is there a sequence of metrics $g_{n}$ on $M$ with diameter $\leq D$ and curvature $\geq-1$ such that $\left(M, g_{n}\right)$ converges to a $k$-dimensional Alexandrov space with $0<k<n$. Of course it would be also interesting if there is a property that is shared by all nonnegatively curved Alexandrov spaces, and which in the case of manifolds is equivalent to saying that the space is rationally elliptic. Alexandrov spaces are more flexible since one can take quotients of non free group actions and in the case positive curvature joins of spaces.

Totaro [2003] posed the problem whether any nonnegatively curved manifold has a good complexification, i.e., is $M$ diffeomorphic to the real points of complex smooth affine variety defined over $\mathbb{R}$ such that the inclusion into the complex variety is a homotopy equivalence. Totoro's work shows that these manifolds share many properties of rationally elliptic manifolds.

The Bott conjecture would also imply that the Eulercharacteristic of a nonnegatively curved manifold is nonnegative and positive only if the odd rational Betti numbers vanish. The former statement is part of the Hopf conjecture.

Conjecture 5.2 (Hopf). A compact nonnegatively (positively) curved manifold has nonnegative respectively positive Eulercharacteristic.

Slightly more modest (and vague) one might ask

QUESTION 5.3. Is there any obstruction that distinguishes the class of simply connected compact manifolds admitting nonnegatively curved metrics from the corresponding class admitting positively curved metrics?

Of course the huge difference in the number of known examples suggests that plenty of such obstructions should exist, but to this day there is not a single dimension where such an obstruction has been found. Closely related is another Hopf conjecture.

Conjecture 5.4 (Hopf). $\mathbb{S}^{2} \times \mathbb{S}^{2}$ does not admit a metric of positive sectional curvature. 
Unlike on $\mathbb{S}^{2} \times \mathbb{S}^{3}$ it is not known whether there is metric on $\mathbb{S}^{2} \times \mathbb{S}^{2}$ with positive curvature almost everywhere. For that reason one could hope that the nonnegatively curved metrics on $\mathbb{S}^{2} \times \mathbb{S}^{2}$ are rather rigid. In fact a partial confirmation of this view was given by Bourguignon, Deschamps, and Sentenac [1972]. They showed that for a product metric on $\mathbb{S}^{2} \times \mathbb{S}^{2}$ without Killing fields any analytical deformation which preserves nonnegative curvature is up to diffeomorphisms given by a deformation through product metrics.

However, one should be careful to expect too much rigidity in this context. The author learned the following observation from Bruce Kleiner. We consider $\mathbb{S}^{2} \times \mathbb{S}^{2}$ endowed with the Müter metric

$$
\left(\mathbb{S}^{2} \times \mathbb{S}^{2}, g\right)=\mathrm{S}^{1} \times \mathrm{S}^{1} \times\{1\} \backslash \mathrm{SO}(3) \times \mathrm{SO}(3) \times \mathrm{SO}(3) / \Delta \mathrm{SO}(3)
$$

where $\mathrm{SO}(3)^{3}$ is endowed with a biinvariant metric. Clearly the metric is of cohomogeneity one, since there is an $\mathrm{SO}(3)$-action from the left on $\mathrm{SO}(3)^{3}$ commuting with the left action of $S^{1} \times S^{1}$. The two singular orbits are given by two 2-dimensional spheres and we let $M_{r e g} \subset \mathbb{S}^{2} \times \mathbb{S}^{2}$ denote the union of all principal orbits. Müter [1987] showed for each point $p \in M_{\text {reg }}$ that there is precisely one zero curvature plane based at $p$. Moreover the plane is tangent to a totally geodesic torus in $M$.

In particular the generic part of the manifold $M_{r e g} \subset \mathbb{S}^{2} \times \mathbb{S}^{2}$ is foliated by totally geodesic flat submanifolds. We now consider a symmetric $(2,0)$ tensor $b$, whose compact support is contained in $M_{\text {reg }}$ and for which $b(v, \cdot)=$ 0 for all $v$ contained in a zero curvature plane. It is then straightforward to check that the foliation of $M_{\text {reg }}$ by totally geodesic flats remains a totally geodesic foliation by flats for all metrics in the family $g(t)=g+t b$. Therefore, the set of zero curvature planes of $(M, g(t))$ contains the set of zero curvature planes in $(M, g(0))$. What is more: the zero curvature planes remain critical points of the sectional curvature. Since the zero curvature planes in $\left(M_{\text {reg }}, g(0)\right)$ form a submanifold of the Grassmannian $\mathrm{Gr}_{2}\left(M_{\text {reg }}\right)$ and the Hessian of the sectional curvature function is nondegenerate transversal to this submanifold, it is clear that $(M, g(t))$ has nonnegative sectional curvature for all small $t$.

This shows that the space of nonnegatively curved metrics of $\mathbb{S}^{2} \times \mathbb{S}^{2}$ is somewhat larger than one would expect at first glance.

One way to give new impulses to the subject is to construct new examples. In this context we pose the following question.

QUESTION 5.5. Are there any positively curved compact Alexandrov spaces satisfying Poincare duality which are not homeomorphic to one of the known positively curved manifolds?

Of course an easy way to check that an Alexandrov space satisfies Poincare duality is to show that the space of directions at each point is 
homeomorphic to a sphere. One could try to look at non free isometric group actions on nonnegatively curved manifolds and ask whether the orbit space is homeomorphic to a manifold without boundary. It would be also interesting to know, whether in special circumstances one can resolve the metric singularities of a positively curved Alexandrov space while keeping positive curvature.

\section{Added in Proof}

One of the most significant developments in the subject took place after this survey was completed. We will briefly explain it here. We recall that a manifold is strictly pointwise quarter pinched if at each point $p \in M$ there is a constant $\kappa(p) \geq 0$ such that for all planes based at $p$ have curvature strictly between $\kappa(p)$ and $4 \kappa(p)$.

Theorem 6.1 (Brendle and Schoen, 2007). For any strictly pointwise quarter pinched manifold $(M, g)$, the normalized Ricci flow evolves $g$ to a limit metric of constant sectional curvature.

We use the notation that we introduced in section 1 in connection with Theorem 1.10. The theorem relies on the following result.

Theorem 6.2 (Böhm and Wilking, 2006). Let $C$ be an $\mathrm{O}(n)$-invariant cone $C$ in the vector space of curvature operators $S_{B}^{2}(\mathfrak{s o}(n))$ with the following properties

- $C$ is invariant under the $O D E \frac{d}{d t} R=R^{2}+R^{\#}$.

- $C$ contains the cone of nonnegative curvature operators or slightly weaker all nonnegative curvature operators of rank 1.

- $C$ is contained in the cone of curvature operators with nonnegative sectional curvature.

Then for any compact manifold $(M, g)$ whose curvature operator is contained in the interior of $C$ at every point $p \in M$, the normalized Ricci flow evolves $g$ to a limit metric of constant sectional curvature.

It actually suffices to assume that the curvature operator of $(M, g)$ is contained in $C$ at all points, and in the interior of $C$ at some point, cf. [Ni and $\mathrm{Wu}, 2006]$.

We should remark that the theorem was not stated like this in [Böhm and Wilking 2006]. However by Theorem 5.1 in that paper it suffices to construct a pinching family with $C(0)=C$. Furthermore, the construction of a pinching family for the cone of nonnegative curvature operators only relied on the above three properties. In other words, one can define a pinching family $C(s)$ with $C(0)=C$ by

$$
C(s):=l_{a(s), b(s)}\left(\left\{R \in C \mid \operatorname{Ric} \geq \frac{\mathrm{scal}}{n} p(s)\right\}\right)
$$


where the parameters $a(s), b(s)$ defining the linear map $l_{a(s), b(s)}: S_{B}^{2}(\mathfrak{s o}(n)) \rightarrow$ $S_{B}^{2}(\mathfrak{s o}(n))$ and $p(s)$ are chosen exactly as in [Böhm and Wilking, 2006].

Sketch of The Proof of TheOrem 6.1.. The most important step was proved independently by Nguyen [2007] and Brendle and Schoen [2007]: Nonnegative isotropic curvature defines a Ricci flow invariant curvature condition. Both proofs are similar. By Hamilton's maximum principle it suffices to show that the cone $C$ of curvature operators with nonnegative isotropic curvature is invariant under the ODE

$$
\frac{d}{d t} R=R^{2}+R^{\#}
$$

The idea is to make use of the second variation formula at a four frame where the isotropic curvature attains a zero - that is one uses the fact that the Hessian of the isotropic curvature function is positive semidefinite. Although the computation is elementary it is quite long and that it succeeds comes close to being a miracle.

Brendle and Schoen then proceed as follows. They consider the condition that a Riemannian manifold crossed with $\mathbb{R}^{2}$ has nonnegative isotropic curvature. It is easy to see that the cone $C$ of curvature operators corresponding to this curvature condition satisfies the hypothesis of Theorem 6.2.

Finally Brendle and Schoen establish that any pointwise quarter pinched manifold $(M, g)$ has the property that $(M, g) \times \mathbb{R}^{2}$ has nonnegative isotropic curvature. This is again a lengthly computation.

REMARK 6.3. Ni and Wolfson [2007] observed that the condition that the manifold crossed with $\mathbb{R}^{2}$ has nonnegative isotropic curvature is equivalent to saying that $(M, g)$ has nonnegative complex sectional curvature. They also give an alternative shorter argument that nonnegative complex curvature is preserved by the Ricci flow. Finally they remark that the statement that quarter pinched manifolds have nonnegative complex curvature was essentially known. In fact Yau and Zheng showed in a different context that a curvature operator with sectional curvature between -4 and -1 has nonpositive complex sectional curvature.

\section{References}

[Abresch (1987)] U. Abresch, Lower curvature bounds, Toponogov's theorem, and bounded topology. II. Ann. Sci. cole Norm. Sup. (4) 20 (1987), no. 3, 475-502.

[Abresch and Meyer (1996)] U. Abresch and W.T. Meyer, A sphere theorem with a pinching constant below $\frac{1}{4}$, J. Diff. Geom. 44 (1996), no. 2, 214-261.

[Aloff and Wallach(1975)] S. Aloff and N. Wallach, An infinite family of 7-manifolds admitting positively curved Riemannian structures, Bull. Amer. Math. Soc. 81(1975), 93-97.

[Bazaikin(1996)] Y.V. Bazaikin, On a certain family of closed 13-dimensional Riemannian manifolds of positive curvature, Sib. Math. J. 37, No. 6 (1996), 1219-1237.

[Belegradek and Kapovitch(2001)] I. Belegradek and V. Kapovitch, Finiteness theorems for nonnegatively curved vector bundles, Duke Math. J. 108 (2001), no. 1, 109-134. 
[Belegradek and Kapovitch(2001)] I. Belegradek and V. Kapovitch, Topological obstructions to nonnegative curvature, Math. Ann. 320 (2001), no. 1, 167-190.

[Belegradek and Kapovitch(2003)] I. Belegradek and V. Kapovitch, Obstructions to nonnegative curvature and rational homotopy theory, J. Amer. Math. Soc. 16 (2003), no. 2, 259-284.

[Bérard Bergery(1975)] L. Bérard Bergery, Sur certaines fibrations d'espaces homogènes riemanniens, Compositio Mathematica 30 (1975), 43-61.

[Bérard Bergery(1976)] L. Bérard Bergery, Les variétés riemanniennes homogènes simplement connexes de dimension impaire à courbure strictement positive, J. Math. pure et appl. $\mathbf{5 5}$ (1976), 47-68.

[Berestovskii and Guijarro(2000)] V.N. Berestovskii and L. Guijarro, A metric characterization of Riemannian submersions, Ann. Global Anal. Geom. 18 (2000), 577-588.

[Berger(1954)] M. Berger, Sur les groupes d'holonomie homogene des varietes a connexion affine et des varietes riemanniennes, Bull. Soc. Math. France 83 (1955), 279-330.

[Berger(1961)] M. Berger, Les varietes riemanniennes homogenes normales simplement connexes a Courbure strictment positive, Ann. Scuola Norm. Sup. Pisa 15 (1961), 191-240.

[Berger(1966)] M. Berger, Trois remarques sur les varietes riemanniennes a courbure strictment positive, C.R.Acad. Sci Paris 262, 1316-1318.

[Böhm and Wilking(2005)] C. Böhm and B. Wilking, Nonnegatively curved manifolds with finite fundamental group admit metrics with positive Ricci curvature, preprint, (2005), to appear in GAFA.

[Böhm and Wilking(2006)] C. Böhm and B. Wilking, Manifolds with positive curvature operators are space forms, to appear in Annals of Math., 15p.

[Borel(1949)] A. Borel, Some remarks about transformation groups on spheres and tori, Bull. Amer. Math. Soc. 55 (1949) 580-587.

[Bourguignon, Deschamps and Sentenac(1972)] J.-P. Bourguignon, A. Deschamps and P. Sentenac, Conjecture de H. Hopf sur les produits de varietes. Ann. Sci. Ecole Norm. Sup. (4) 5 (1972), 277-302.

[Bredon(1972)] G. Bredon, Introduction to compact transformation groups, Pure and Applied Mathematics, Vol. 46. Academic Press, New York-London, 1972.

[Brendle and Schoen(2007)] S. Brendle and R. Schoen, 1/4-pinched manifolds are space forms, preprint, $25 \mathrm{p}$.

[Cheeger(1973)] J. Cheeger, Some examples of manifolds of nonnegative curvature, J. Differential Geom. 8 (1973), 623-628.

[Cheeger and Ebin(1975)] J. Cheeger and D. Ebin, Comparison theorems in Riemannian geometry, North-Holland Mathematical Library, Vol. 9. New York, 1975.

[Cheeger and Gromoll(1971)] J. Cheeger and D. Gromoll, The splitting theorem for manifolds of nonnegative Ricci curvature. J. Differential Geometry 6 (1971/72), 119-128.

[Cheeger and Gromoll(1972)] J. Cheeger and D. Gromoll, On the structure of complete manifolds of nonnegative sectional curvature, Ann. of Math. 96 (1972), 413-443.

[Chen(1991)] H. Chen, Pointwise 1/4-pinched 4-manifolds, Ann. Global Geom. 9 (1991), $161-176$.

[Chen and Tian(2006)] X. Chen and G. Tian Ricci flow on Kähler-Einstein manifolds, Duke Math. J. 131 (2006), no. 1, 17-73.

[Chow(1991)] B. Chow, The Ricci flow on the 2-sphere, J. Differential Geom. 33 (1991), 325-334.

[Chow and $\mathrm{Wu}(1991)]$ B. Chow and L.-F. Wu, The Ricci flow on compact 2-orbifolds with curvature negative somewhere, Comm. Pure. Appl. Math. 44 (1991), 275-286.

[Chow and Yang(1989)] B. Chow and D. Yang, Rigidity of nonnegatively curved quaternionic-Kähler manifolds, J. Differntial Geom. 29 (1989), 361-372.

[Dessai(2005)] A. Dessai, Characteristic numbers of positively curved Spin-manifolds with symmetry. Proc. Amer. Math. Soc. 133 (2005), no. 12, 3657-3661. 
[Dessai and Wilking(2004)] A. Dessai and B. Wilking, Torus actions on homotopy complex projective spaces, Math. Z., 247 (2004), 505-511.

[Eschenburg(1982)] J. H. Eschenburg, New examples of manifolds with strictly positive curvature, Invent. Math 66 (1982), 469-480.

[Eschenburg(1984)] J. H. Eschenburg, Freie isometrische Aktionen auf kompakten LieGruppen mit positiv gekrümmten Orbiträumen, Schriftenr. Math. Inst. Univ. Münster 32 (1984).

[Eschenburg(1992)] J.H. Eschenburg, Inhomogeneous spaces of positive curvature, Diff. Geom. and its App. 2 (1992), 123-132.

[Fang and Rong(2005)] F. Fang and X. Rong, Homeomorphism classification of positively curved manifolds with almost maximal symmetry rank, Math. Ann. 332, (2005), 81-101.

[Fukaya and Yamaguchi(1992)] K. Fukaya and T. Yamaguchi, The fundamental groups of almost nonnegatively curved manifolds, Ann. of Math. 136 (1992), 253-333.

[Gromoll and Grove(1987)] D. Gromoll and K. Grove, A generalization of Berger's rigidity theorem for positively curved manifolds, Ann. Sci. Ec. Norm. Super., IV. Ser. 20 (1987), 227-239.

[Gromoll and Grove(1988)] D. Gromoll and K. Grove, The low-dimensional metric foliations of Euclidean spheres, J. Differ. Geom. 28 (1988), 143-156.

[Gromoll and Meyer(1969)] D. Gromoll and W. T. Meyer, On complete open manifolds of positive curvature, Ann. of Math. (2) 90 (1969), 75-90.

[Gromoll and Meyer(1974)] D. Gromoll and W. T. Meyer, An exotic sphere with nonnegative sectional curvature, Ann. of Math. 100 (1974), 401-406.

[Gromov(1978)] M. Gromov, Almost flat manifolds. J. Differential Geom. 13 (1978), no. 2, 231-241.

[Gromov(1981)] M. Gromov, Curvature diameter and Betti numbers, Comment. Math. Helv. 56 (1981), 179-195.

[Gromov(1981)] M. Gromov, Curvature, diameter and Betti numbers, Comment. Math. Helv. 56 (1981), no. 2, 179-195.

[Gromov(1986)] M. Gromov, Partial differential relations. Ergebnisse der Mathematik und ihrer Grenzgebiete (3), 9. Springer-Verlag, Berlin, 1986. x+363 pp.

[Grove and Halperin(1987)] K. Grove and S. Halperin, Dupin hypersurfaces, group actions and the double mapping cylinder, J. Differential Geom. 26 (1987), no. 3, 429-459.

[Grove et al.(1974)] K. Grove, H. Karcher and E.A. Ruh, Group actions and curvature, Invent. Math. 23 (1974), 31-48.

[Grove and Searle(1994)] K. Grove and C. Searle, Positively curved manifold of maximal symmetry rank J. Pure Appl. Algebra 91 (1994), 137-142.

[Grove and Searle(1997)] K. Grove and C. Searle, Differential topological restrictions by curvature and symmetries J. Differ. Geom. 47 (1997), 530-559.

[Grove and Shiohama(1977)] K. Grove and K. Shiohama, A generalized sphere theorem, Ann. Math. (2) 106 (1977), 201-211.

[Grove et al. (2006)] K. Grove, L. Verdiani, B. Wilking and W. Ziller, Non-negative curvature obstructions in cohomogeneity one and the Kervaire spheres, Ann. Sc. Norm. Super. Pisa Cl. Sci. (5) 5 (2006), no. 2, 159-170.

[Grove and Wilhelm(1997)] K. Grove and F. Wilhelm, Metric contraints on exotic spheres via Alexandrov geometry, J. Reine Angew. Math. 487 (1997), 201-217.

[Grove, Wilking and Ziller(2005)] K. Grove, B. Wilking and W. Ziller, Positively curved Cohomogeneity one manifolds and 3 Sasakian geometry, preprint, 79p, to appear in J. Differ. Geom.

[Grove and Ziller(2000)] K. Grove and W. Ziller, Curvature and symmetry of Milnor spheres, Ann. of Math. 152 (2000), 331-367.

[Guijarro(2000)] L. Guijarro, Improving the metric in an open manifold with nonnegative curvature, Proc. Amer. Math. Soc. 126 (1998), no. 5, 1541-1545. 
[Guijarro(2000)] L. Guijarro, On the metric structure of open manifolds with nonnegative curvature, Pacific J. Math. 196 (2000), 429-444.

[Guijarro and Pertersen(1997)] L. Guijarro and P. Petersen, Rigidity in non-negative curvature, Ann. Sci. cole Norm. Sup. (4) 30 (1997), no. 5, 595-603.

[Gursky and LeBrun(1999)] M. Gursky and C. LeBrun, On Einstein manifolds of positive sectional curvature, Ann. Global Anal. Geom. 17 (1999), no. 4, 315-328.

[Hamilton(1982)] R. Hamilton, Three-manifolds with positive Ricci curvature, J. Differential Geom. 17 (1982), 255-306.

[Hamilton(1986)] R. Hamilton, Four-manifolds with positive curvature operator, J. Differential Geom. 24 (1986), 153-179.

[Hamilton(1988)] R. Hamilton, The Ricci flow on surfaces, Mathematics and general relativity, 237-262, Contemp. Math. 71, Amer. Math. Soc. (1988).

[Hamilton(1995)] R. Hamilton, The formation of singularities in the Ricci flow, Surveys in Differential Geometry, Vol II, International Press (1995), 7-136.

[Hitchin(1996)] N. Hitchin, A new family of Einstein metrics, Manifolds and geometry (Pisa, 1993), 190-222, Sympos. Math., XXXVI, Cambridge Univ. Press, Cambridge, 1996.

[Hsiang and Kleiner(1989)] W. Y. Hsiang and B. Kleiner, On the topology of positively curved manifold with symmetry, J. Differential Geom. 30 (1989), 615-621.

[Huisken(1985)] G. Huisken, Ricci deformation on the metric on a Riemannian manifold, J. Differential Geom. 21 (1985), 47-62.

[Im Hof and Ruh (1975)] H.C. Im Hof and E. Ruh, An equivariant pinching theorem, Comment. Math. Helv. 50 (1975), 389-401.

[Kapovitch, Petrunin and Tuschman (2005)] V. Kapovitch, A. Petrunin and W. Tuschmann, Nil-potency, Almost Nonnegative curvature and the gradient push, preprint 2005, 24 p, to appear in Ann. of Math.

[Kleiner(1990)] B. Kleiner, Riemannian four-manifolds with nonnegative curvature and continuous symmetry, Thesis, Univ. Of California, Berkeley, (1990).

[Micallef and Moore(1988)] M. Micallef and J.D. Moore, Minimal two-spheres and the topology of manifolds with positive curvature on totally isotropic two-planes, Ann. of Math. (2) 127 (1988), 199-227.

[Molino(1988)] P. Molino, Riemannian foliations, Birkhäuser Boston, Inc., Boston, MA, 1988. xii+339 pp.

[Mori(1979)] S. Mori, Projective manifolds with ample tangent bundles, Ann. of Math. (2) 110 (1979), no. 3, 593-606.

[Müter(1987)] M. Müter, Krümmungserhöhende Deformationen mittels Gruppenaktionen, Thesis, (1987), Math. Institut Münster.

[Myers and Steenrod(1939)] S.B. Myers and N.E. Steenrod, The group of isometries of a Riemannian manifold, Ann. of Math. 40 (1939), 400-416.

[Nguyen(2007)] . Nguyen, Invariant curvature cones and the Ricci flow, preprint $16 \mathrm{p}$.

$[\mathrm{Ni}(2004)] \mathrm{L} . \mathrm{Ni}$, Ricci flow and nonnegativity of sectional curvature, Math. Research Letters 11, 883-904.

[Ni and Wolfson (2007)] L. Ni and J. Wolfson, Positive Complex Sectional Curvature, Ricci Flow and the Differential Sphere Theorem, preprint, (2007), 15p.

[Ni and $\mathrm{Wu}(2006)] \mathrm{L} . \mathrm{Ni}$ and $\mathrm{B}$. Wu, Complete manifolds with nonnegative curvature operator, preprint, (2006), $7 \mathrm{p}$.

[O'Neill(1966)] B. O'Neill, The fundamental equations of a submersion, Michigan Math. J. 13 (1966), 459-469.

[Özaydin and Walschap(1994)] M. Özaydin and G. Walschap, Vector bundles with no soul. Proc. Amer. Math. Soc. 120 (1994), no. 2, 565-567.

[Perelman(1994)] G. Perelman, Proof of the soul conjecture of Cheeger and Gromoll, J. Differential Geom. 40 (1994), 209-212. 
[Perelman(2003)] G. Perelman, The entropy formula for the Ricci flow and its geometric applications, arXiv:math.DG/0307245.

[Petersen(2006)] P. Petersen, Riemannian geometry, second edition. Graduate Texts in Mathematics, 171. Springer, New York, 2006 Springer.

[Petersen(2006)] P. Petersen and F. Wilhelm, Examples of Riemannian manifolds with positive curvature almost everywhere, Geom. Topol. 3 (1999), 331-367.

[Rigas(1985)] A. Rigas, Reprentantes de fibrados sobre esferas, Atas da VI Escola de Geometria Diferencial, IMPA, 1985, 129-140.

[Rong(2002)] X. Rong Positively curved manifolds with almost maximal symmetry rank, Geom. Dedicata 95 (2002), 157-182.

[Schwachhöfer and Tuschmann(2004)] L. Schwachöfer and W. Tuschmann, Metrics of positive Ricci curvature on quotient spaces, Math. Ann. 330 (2004), 59-91.

[Searle and Yang(1994)] C. Searle and D.G. Yang On the topology of non-negatively curved 4-manifolds with continuous symmetry, Duke Math. J. 74, 547-556.

[Shankar(1998)] K. Shankar, On the fundamental groups of positively curved manifolds, J. Differential Geom. 49 (1998), no. 1, 179-182.

[Sharafutdinov(1979)] V.A. Sharafutdinov, Convex sets in a manifold of nonnegative curvature. (Russian) Mat. Zametki 26 (1979), no. 1, 129-136, 159.

[Siu and Yau(1980)] Y. Siu and S.T. Yau, Compact Kähler manifolds of positive bisectional curvature, Invent. Math. 59 (1980), no. 2, 189-204.

[Strake, 1988] M. Strake, A splitting theorem for open nonnegatively curved manifolds, Manuscripta Math. 61 (1988), no. 3, 315-325.

[Sugimoto and Shiohama(1971)] M. Sugimoto and K. Shiohama, On the differentiable pinching problem, Math. Ann. 195 (1971), 1-16.

[Suyama(1991)] Y. Suyama,Differentiable sphere theorem by curvature pinching, J. Math. Soc. Japan 43, No. 3 (1991), 527-553.

[Suyama(1995)] Y. Suyama, A differentiable sphere theorem by curvature pinching II, Tôhoku Math. J. 47 (1995), 15-29.

[Totaro, 2003] B. Totaro, Complexifications of nonnegatively curved manifolds, J. Eur. Math. Soc. (JEMS) 5 (2003), no. 1, 69-94.

[Verdiani(2003)] L. Verdiani, Cohomogeneity one manifolds of even dimension with strictly positive sectional curvature, J. Differential Geom. 68 (2004), no. 1, 31-72.

[Walschap(1988)] G. Walschap, Nonnegatively curved manifolds with souls of codimension 2, J. Differential Geom. 27 (1988), no. 3, 525-537.

[Wallach(1972)] N. Wallach, Compact homogeneous Riemannian manifolds with strictly positive curvature, Ann. of Math. 96 (1972), 277-295.

[Weiss(1993)] M. Weiss, Pinching and concordance theory, J. Differential Geom. 38 (1993), 387-416.

[Wilhelm(1996)] F. Wilhelm, The radius rigidity theorem for manifolds of positive curvature, J. Differ. Geom. 44 (1996), 634-665.

[Wilking(2000)] B. Wilking, On fundamental groups of manifolds of nonnegative curvature, Differen. Geom. Appl. 13 (2000).

[Wilking(2001)] B. Wilking, Index parity of closed geodesics and rigidity of Hopf fibrations, Invent. Math. 144 (2001), 281-295.

[Wilking(2002)] B. Wilking, Manifolds with positive sectional curvature almost everywhere. Invent. Math. 148 (2002), no. 1, 117-141.

[Wilking(2003)] B. Wilking, Torus actions on manifolds of positive sectional curvature, Acta Mathematica, 191 (2003), 259-297.

[Wilking(2005)] B. Wilking, A duality theorem for singular Riemannian foliations in nonnegative curvature, preprint, 2005, to appear in GAFA. 
[Wilking(2006)] B. Wilking, Positively curved manifolds with symmetry, Ann. of Math. 163 (2006), no. 2, 607-668.

[Yim(1990)] J.-W. Yim, Space of souls in a complete open manifold of nonnegative curvature, J. Differential Geom. 32 (1990), no. 2, 429-455.

[Zizhou(2002)] T. Zizhou, A note on the cohomological 1/4-Pinching theorem, Proc. Amer. Math. Soc. 130 (2002), 577-578.

Universität MÜnster, Einsteinstrasse 62, 48149 MÜnster, Germany

E-mail address: wilking@math.uni-muenster.de 